\title{
Structural analysis of 3'UTRs in insect flaviviruses reveals novel determinant of sfRNA biogenesis and provides new insights into flavivirus evolution
}

Andrii Slonchak ${ }^{1, \#}$, Rhys Parry ${ }^{1}$, Brody Pullinger ${ }^{1}$, Julian D.J. Sng ${ }^{1}$, Xiaohui Wang ${ }^{1}$, Teresa F Buck $^{1,2}$, Francisco J Torres ${ }^{1}$, Jessica J Harrison'1, Agathe M G Colmant ${ }^{1}$, Jody HobsonPeters $^{1,3}$, Roy A Hall ${ }^{1,3}$, Andrew Tuplin ${ }^{4}$, Alexander A Khromykh ${ }^{1,3, \#}$

${ }^{1}$ School of Chemistry and Molecular Biosciences, University of Queensland, Brisbane, QLD, Australia

${ }^{2}$ Current affiliation: Institute for Medical and Marine Biotechnology, University of Lübeck, Lübeck, Germany

${ }^{3}$ Australian Infectious Diseases Research Centre, Global Virus Network Centre of Excellence, Brisbane, QLD, Australia

${ }^{4}$ School of Molecular and Cellular Biology, University of Leeds, Leeds, U.K.

\# corresponding authors: a.khromykh@uq.edu.au; a.slonchak@uq.edu.au 


\section{Abstract}

Insect-specific flaviviruses (ISFs) circulate in nature due to vertical transmission in mosquitoes and do not infect vertebrates. ISFs include two distinct lineages - classical ISFs (cISFs) that evolved independently and dual host associated ISFs (dISFs) that are proposed to diverge from mosquito-borne flaviviruses (MBFs). Compared to pathogenic flaviviruses, ISFs are relatively poorly studied, and their molecular biology remains largely unexplored. In this study we focused on the characterisation of ISF 3'UTRs and their ability to produce subgenomic flaviviral RNAs - noncoding viral RNAs that are known as important determinants of transmission and replication of pathogenetic flaviviruses. We demonstrated that cISFs and dISFs produce sfRNAs by employing a highly conserved mechanism of resistance to degradation by the cellular 5'-3' exoribonuclease XRN1. We determined the secondary structures of complete 3'UTRs and experimentally identified structured RNA elements that resist degradation by XRN1 (xrRNAs) in divergent representatives of clSF and dISF clades. We discovered a novel class of xrRNAs in dISFs and identified structurally divergent xrRNA in Anopheles-associated clSFs. Phylogenetic analyses based on sequences and secondary structures of xrRNAs and complete 3'UTRs reveal that xrRNAs of cISFs and MBFs/dISFs evolved from a common xrRNA ancestor similar to the xrRNA of Anopheles-associated cISFs. Additionally, we found that duplications of xrRNAs occurred independently in ISF and MBF clades. Using ISF mutants deficient in the production of sfRNAs, we found that individual sfRNAs of ISFs have redundant functions. We conclude that duplicated xrRNAs were selected in the evolution of flaviviruses to ensure that sfRNA is produced if one of the xrRNAs lose XRN1 resistance due to mutations or misfolding. 


\section{Introduction}

Flaviviruses are generally known as important human pathogens capable of causing severe diseases and unpredictable outbreaks. However, the flavivirus genus is very diverse and includes viruses that cannot replicate in vertebrates. This group of viruses, known as insect-specific flaviviruses (ISF), recently attracted significant attention due to their ability to inhibit replication of pathogenic flaviviruses in co-infected mosquitoes ${ }^{1}$ and their potential use as a safe recombinant vaccine platform ${ }^{2}$. The Flavivirus genus can be divided into the following ecological groups: mosquito-borne flaviviruses (MBFs), which circulate between mosquito and vertebrate (avian, equine or human) hosts; tick-borne flaviviruses (TBFs) that are maintained in nature in tick-vertebrate cycle $^{3}$; viruses that only infect vertebrates and are thought to be transmitted horizontally ${ }^{4}$ (no known vector flaviviruses, NKVFs) and insectspecific flaviviruses (ISFs) that infect mosquitoes or sand flies and are believed to circulate predominantly via vertical transmission ${ }^{5}$. ISFs include two phylogenetically distinct lineages - (i) lineage I or classical ISFs (cISFs) that independently diverged from an ancient flavivirus ancestor and (ii) lineage II or dual host associated ISFs (dISFs) that are believed to be evolved from MBFs that lost the ability to infect vertebrates ${ }^{5,6}$.

To replicate in diverse hosts, flaviviruses have evolved multiple mechanisms to counteract, subvert and evade host antiviral responses. One of them is by producing noncoding viral RNA named subgenomic flaviviral RNA (sfRNA) (reviewed in ${ }^{7}$ ). In infected cells, viral genomic RNA is subjected to degradation by the host 5'-3' exoribonuclease XRN1. XRN1 is a highly processive enzyme with a helicase activity, which can unwind and fully digest virtually any $\mathrm{RNA}^{8}$. However, flaviviruses contain uniquely folded RNA elements in their 3'UTRs that can halt the progression of $X R N 1{ }^{9}$. Stalling of $X R N 1$ prevents complete degradation of viral genomic RNA and results in accumulation of 3'UTR-derived sfRNAs in the infected cells ${ }^{10}$ (Supplementary figure 1A). Multiple studies have demonstrated that 
sfRNAs facilitate viral replication and pathogenesis by inhibiting IFN response in vertebrates ${ }^{11-15}$ and $\mathrm{RNAi}^{16,17}$ or apoptosis ${ }^{18}$ in mosquitoes. However, all these results were obtained using vertebrate-infecting dual host flaviviruses, while biogenesis and functions of sfRNAs in ISFs remain poorly characterized. To date, production of sfRNA was reported for only one ISF - cell fusing agent virus (CFAV) ${ }^{19}$.

XRN1-resistant elements are believed to have conserved secondary and tertiary structures (Supplementary figure 1B, C) within each clade of flaviviruses ${ }^{7}$. Commonly, all flavivirus xrRNAs characterized to date are formed by the stem-loops (SL) that contain a three-way junction between three RNA helices (P1, P2 and P3) and a pseudoknot (PK) formed by the terminal loop (L2) of P2 helix (Supplementary figure 1C). In addition, xrRNAs contain a small pseudoknot (sPK) within a junction between $\mathrm{P} 1$ and $\mathrm{P} 3$ helices (Supplementary figure 1C). The pseudoknots and non-canonical interactions within xrRNAs determine their unique tertiary conformation in which the 5'-end of the RNA passes through a ring-like structure ${ }^{20,21}$ (Supplementary figure 1B). This unique fold determines the resistance of $\mathrm{xrRNAs}$ to $\mathrm{XRN1}$ as the RNA ring creates a roadblock for the progression of the enzyme $^{22}$ (Supplementary figure 1A). To date, secondary structures have been experimentally determined for xrRNAs of MBFs ${ }^{9,23,24}, \mathrm{TBFs}^{19}, \mathrm{NKVFs}^{19,25}$ and $\mathrm{cISF}^{19 F A V}{ }^{19}$. In addition, crystal structures have been solved for three MBF $\operatorname{xrRNAs}^{20,21}$ and $x \mathrm{rRNA}$ of NKVF Tamana Bat virus ${ }^{25}$. Based on the conserved structural elements, xrRNAs are classified into two classes - class 1 xrRNAs occur in MBF, while class 2 xrRNAs occur in TBFs and NKVFs ${ }^{19}$. Within class 1, two additional subclasses can be distinguished subclass 1 includes the typical MBF xrRNAs that contain 5-nt P1 helix, 5-nt pseudoknot and conserved unpaired nucleotide between P2 and P3 ${ }^{20}$ (Supplementary Figure 1C). Subclass 1b xrRNAs were identified in NKVF Tamana Bat virus (TABV) and contain 3-4-nt P1 helix, often with non-canonical A-C base pairing at the base of the helix, 3-nt pseudoknot and no 
unpaired bases between P2 and P325,26 (Supplementary Figure 1C). Based on covariance models and/or sequence alignments, dISFs were predicted to contain class 1 a $\mathrm{xrRNAs}^{27}$, although these predictions have not been experimentally validated.

3'UTRs of MBFs typically contain duplicated SL-based xrRNAs followed by two dumbbell (DB) structures and a 3'-terminal stem-loop (3'SL) ${ }^{27}$. The duplicated SLs give rise to two sfRNAs of different length ${ }^{28}$. In addition, two smaller sfRNAs are produced by MBFs at the dumbbell structures. However, the biogenesis mechanism of these RNAs is unclear as dumbbells have been recently shown to lack XRN1 resistance ${ }^{29}$. Currently, it is not fully understood why MBFs acquired additional xrRNAs and whether different length isoforms of sfRNAs have redundant or specialised functions. One study demonstrated that Dengue virus 2 (DENV2) accumulates mutations in the individual xrRNAs and switch between the production of longer and shorter sfRNAs when switching hosts ${ }^{13}$. This suggests that duplication of the structural elements within 3'UTR represents an adaptation to the dual host life cycle, and individual sfRNA species are specifically adapted to function in different hosts. Although the production of sfRNAs by dISFs is yet to be shown, their 3'UTRs were predicted to contain a single SL and a single dumbbell followed by $3^{\prime} \mathrm{SL}^{27}$. As dISFs are believed to evolve from MBFs that lost the ability to infect vertebrates, it is possible that they lost duplicated 3'UTR elements due to transition to a single host life cycle with no need for switching sfRNA production while adapting to different hosts. Two previous attempts to predict the structure of cISF 3'UTRs did not reveal high-order secondary structures, identifying only short inverted repeats ${ }^{27,30}$. However, more recent work suggested that dISFs may contain class $1 \mathrm{~b}$ xrRNAs, similar to those identified in TBAV 26.

The growing interest in ISFs as potential biocontrol agents and as vaccine platform and the lack of knowledge about 3'UTR structure and production of sfRNA prompted us to perform thorough structural and functional analysis of the 3'UTRs in representatives of cISFs 
and dISFs. We demonstrated that dISFs and cISFs, including the most phylogenetically distant Anopheles-associated ISFs, generate sfRNAs by employing XRN1-resistant structured RNA elements. Using chemical probing for RNA structure, we for the first time, experimentally determined the secondary structures of the complete 3'UTRs of cISFs and dISFs. We discovered that dISFs contain a novel class of XRN1-resistant elements that complement class 1a xrRNAs for the robust production of sfRNAs. We also found that clSFs and the dISF BinJV contain multiple copies of xrRNAs. However, Anopheles-associated cISFs contain only a single XRN1-resistant structure, which has features of both class 1a and class $1 \mathrm{~b}$ structures and is ancestral to xrRNAs of ISFs and MBFs. Furthermore, our structurebased phylogenetic analysis of 3'UTRs revealed that the evolution of flaviviruses involved several xrRNA duplication events that occurred independently in cISF, MBF and dISF clades, indicating their significant benefit for viral fitness. Finally, we used ISF mutants with impaired functions of individual or all xrRNAs to demonstrate that different sfRNA species likely have redundant functions in ISFs. Our data indicate that duplication of xrRNAs and the acquisition of novel XRN1-resistant structures serve to ensure robust sfRNA production and its protection from potentially damaging impacts of inadvertent mutations.

\section{Results}

\section{Classical and dual host associated ISFs produce sfRNAs via XRN1 resistance} mechanism

To determine whether phylogenetically divergent cISFs and dISFs are capable of sfRNA production, we performed a Northern blot of total RNA isolated from C6/36 cells infected with Aedes-associated cISF Parramatta River Virus (PaRV) ${ }^{31}$, Culex-associated cISF Palm Creek virus (PCV) ${ }^{32}$ and dISFs Binjari virus (BinJV) ${ }^{2,33}$ and Hidden valley virus $(\mathrm{HVV})^{33}$. To test for production of sfRNA by Anopheles-associated Karumba virus (KRBV), 
which is unable to replicate in all tested laboratory cell lines ${ }^{34}$, RNA from virus-positive mosquitoes collected in the field was used. All tested viruses were found to produce sfRNAs (Fig 1A). PCV and BinJV produced three sfRNAs of different length. In contrast, HVV produced two sfRNAs, PaRV produced two major sfRNAs species and potentially one less abundant sfRNA, while Anopheles-associated flavivirus KRBV produced only one sfRNA (Fig 1A). To further identify the structural determinants of sfRNA biogenesis in ISFs, we then determined the 5'-ends of identified sfRNAs using the previously described RNA ligationmediated RT-PCR method ${ }^{13}$. We were able to identify 5'-ends of PaRV, PCV and BinJV sfRNAs and map them to viral 3'UTRs (Supplementary figure 2). We did not obtain an amplification product for KRBV sfRNA, which was likely due to the low viral load in mosquito field samples.

To elucidate if XRN1 is responsible for sfRNA biogenesis in ISFs, the effect of XRN1 knock-down on the production of sfRNA by PaRV, PCV and BinJV were assessed. Transfection of dsRNA against XRN1 reduced production of sfRNAs by PaRV and PCV. It also resulted in decreased accumulation of BinJV sfRNA-1 and -2 . These results show that sfRNAs in CISF and major sfRNA species in dISFs are produced via incomplete digestion of 3'UTRs by XRN1. To assess if XRN1 resistance in ISFs is determined by structured RNA elements, the ability of XRN1 to digest folded and denatured viral 3'UTRs into sfRNA in vitro was tested. Treatment with $\mathrm{XRN1}$ resulted in partial degradation of refolded in vitro transcribed 3'UTRs of PaRV, PCV, BinJV and KRBV (Fig 1C) and production of sfRNAs that had the same length as those detected in infection (Fig 1A). However, 3'UTRs of all examined viruses became highly susceptible to complete degradation by XRN1 if RNA was denatured (Fig 1C). This indicates that XRN1 resistance in clSFs and dISFs is determined by structured RNA. The in vitro XRN1 digestion assay also shows that XRN1 is responsible for the 
production of sfRNA by KRBV, which we could not use in the knock-down experiment due to the lack of virus replication in cell lines.

In conclusion, we demonstrated that cISFs and dISFs produce sfRNAs due to the presence of structured XRN1-resistant RNA elements in their 3'UTRs. We also found that all tested ISFs except KRBV produce multiple sfRNA species, which infers the existence of several XRN1 resistant structures in their 3 UTRs. We also identified 5'-ends of PaRV, PCV, and BinJV sfRNAs for further mapping to the structured elements in viral 3'UTRs (putative xrRNAs).

\section{Dual host associated ISFs contain a novel class of XRN1-resistant RNA elements}

To determine the secondary structure of BinJV 3'UTR and identify structural determinants of sfRNA biogenesis in dISFs, we employed selective 2 '-hydroxyl acylation analysed by primer extension (SHAPE). This method identifies paired and unpaired nucleotides in the in vitro transcribed and refolded $\mathrm{RNA}^{35}$. The base pairing information is then used to guide the computational folding of RNA structures. SHAPE results demonstrated that the BinJV 3'UTR contains two PK-forming SLs with 3-way junctions, a single copy of dumbbell element and a canonical 3'SL with a preceding short hairpin (Fig 2A). PK-forming stem-loop elements of BinJV had features of class 1a xrRNAs such as unpaired $\mathrm{C}$ nucleotide in P2-P3 junction, conserved GU upstream of P1 stem that formed a small PK with nucleotides between P3 and P1, and 5-6nt long range PK formed by the L2 loop (Fig 2B, C). Therefore they structurally fit into class 1a of xrRNAs typical for MBFs and have significant sequence and structural homology with them (Fig 2C). Looking at BinJV xrRNAs (Fig 2B), we will expect a $U-A-U$ base triple between $U 4$ and A22-U43 (the numbers are for xrRNA1) (Fig 2C), and the base-pairs between A2-G3 and C42-U43 into the three-way junction (sPK 
Fig 2B, C). Therefore we can be confident that there will be a ring of $14 \mathrm{nt}$ that will form, and this almost certainly will be when the top of P3 rearranges to cause A38 to extrude out from the helix and interact with U52 in a long-range reverse-Watson-Crick pair. These structural features were seen in the Zika xrRNA ${ }^{20}$, and the homology between the Zika sequence and secondary structure and the BinJV sequence and secondary structure (Fig 2C) means that both BinJV SLs can be confidently predicted to fold almost identical to the Zika xrRNA1. Therefore we can say with confidence that they form the ring-like structure required for XRN1 resistance. Moreover, BinJV SLs are located at positions corresponding to the 5'-ends of identified sfRNAs (Supplementary figure 2, Fig 2A). In addition, disruption of PK interaction in SLI and SLII by mutations greatly reduced the production of sfRNA1 and sfRNA2, respectively, in in vitro XRN1 digestion assay (Fig 2D). This directly confirmed the XRN1 resistant nature of these elements and the requirement of pseudoknot interactions for their resistance. Therefore, we concluded that BinJV contains two copies of class 1a xrRNAs.

Further examining the structure of BinJV 3'UTR we noticed that small stem-loops CS3 and RCS3 downstream of each xrRNA SL also formed pseudoknots that we named novel pseudoknots nPK 1 and nPK2 (Fig 1A). Although similar short stem-loops are present in all MBFs, they are not involved in PK interactions in any of the flaviviruses for which structural data on the 3'UTR is available ${ }^{9,23}$ and have not been predicted to form PKs. Interestingly, in contrast to other flaviviruses $9,10,19,23$, disruption of PK1 in xrRNA1 of BinJV by mutations did not abolish XRN1-resistance completely (Fig 1D). Hence we hypothesised that nPKs might stabilise xrRNAs or confer additional XRN1 resistance. To test this hypothesis, we assessed the effects of mutations that disrupt nucleotide pairing in $\mathrm{nPK} 1$ and $\mathrm{nPK} 2$ on the production of BinJV sfRNAs in vitro. Notably, disruption of $\mathrm{nPK} 1$ significantly reduced the generation of sfRNA-1 (Fig 2D, E). We also observed decreased production of sfRNA2 after mutation in $\mathrm{nPK} 2$, although the difference was not statistically significant. This, as well as the complete 
disappearance of sfRNA-2 upon PK2 disruption, was likely due to the initially very low level of this sfRNA produced from the WT sequence in vitro (Fig 2D, E). These results indicate that nPKs contribute to the production of sfRNAs from BinJV 3'UTR and comprise novel xrRNAs formed by small stem-loops downstream of the canonical xrRNAs. They confer XRN1 resistance while lacking conserved structural elements of the canonical flaviviral xrRNAs such as three-way junction and small PK, representing a separate class of xrRNAs.

To elucidate whether n-xrRNAs are unique for BinJV or represent a more common for dISFs feature, we first used IPknot ${ }^{36}$ software to predict PKs in 3'UTRs of all dISFs for which complete 3'UTR sequences were available. The analysis demonstrated that all dISFs (Supplementary figure 3A) except NHUV (Supplementary figure 3B) contain a putative pseudoknot formed by small stem-loops downstream of the canonical xrRNAs and potentially have novel xrRNAs. We then performed structure based sequence alignment of dISF xrRNAs-n-xrRNA regions (Supplementary figure 3C), built a covariance model ${ }^{37}$ and used it to identify the consensus secondary structure (Fig 2F). The covariance analysis demonstrated that canonical xrRNAs had both high sequence and high structure homologies with two conserved and at least three covarying nucleotide pairs (preserve complementary if vary) in the PK region (Fig 2F). Novel xrRNAs were found to lack sequence conservancy but maintained a conserved structure with at least three covarying nucleotides in the stem. Importantly, novel PKs were shown to be structurally conserved with four highly (>97\%) covarying nucleotide pairs. This indicates that the ability to form novel pseudoknots is conserved among dISFs. To validate the predicted nPKs in other dISF representative, we performed SHAPE on the fragment of the 3'UTR of HVV, which demonstrated nucleotide pairing between the loop region of the hairpin element and a downstream sequence (Fig. $2 \mathrm{G})$, confirming that novel xrRNAs are not unique to BinJV and further supporting the consensus structure of novel xrRNAs generated based on the covariance model. 
Therefore, we demonstrated that dISFs contain a novel class of xrRNAs evolved from the conserved stem-loop elements located downstream of the canonical xrRNAs. In the current classification of xrRNA types, they will be class 3 xrRNAs. These xrRNAs have very simple organisation, lacking a three-way junction and bearing only one PK while still forming an exonuclease-resistant ring-like fold. They occur commonly in dISFs, but not MBFs and complement major xrRNAs conferring additional XRN1 resistance to produce sfRNAs. In addition, the secondary structure of dISF 3'UTR was experimentally determined here for the first time, demonstrating that this group of flaviviruses can contain duplicated SLs.

\section{Classical ISFs PaRV and PCV contain multiple copies of class 1b xrRNAs}

To identify structural determinants of sfRNA biogenesis in clSFs, we determined the secondary structure of 3'UTRs of PaRV and PCV using SHAPE. SHAPE-assisted folding of PaRV 3'UTR revealed that it contains five stem-loop elements, four of which form pseudoknots and are followed by one or two small stem-loops similar to CS3 and RCS3 elements of MBFs/dISFs (Fig 3A). PaRV 3'UTR also contained a 3'-terminal SL similar to all other flaviviruses while having no dumbbell elements (Fig 3A). PK-forming stem-loops in PaRV 3'UTR had properties of class $1 \mathrm{~b}$ xrRNAs and generally corresponded to the recently predicted structures ${ }^{26}$ with minor differences. Two of them (SLI and SLIII) were mapped to the 5'-termini of the PaRV sfRNA-1 and sfRNA-3 (Fig 3A). Although we were unable to determine the exact 5'-end of less abundant sfRNA-2, the length of this RNA infers that it should be produced at SLII in PaRV 3'UTR (Fig 1A, 3A). Notably, neither Northern blot of RNA from infected cells (Fig 1A) nor in vitro XRN1 digestion (Fig 1C) revealed production of sfRNA from SLIV, suggesting that SLIV does not represent a functional xrRNA despite the apparent structural similarities. From comparing PaRV SLI, II, and III (Fig 1B), we can conclude that $x r R N A 2$ acquired a mutation of $U$ to $A$ at the first position of $L 2$, which created 
additional base pair in the P3 helix, extending its length. Given that the P3 helix is involved in forming the RNA ring, this should result in the structure that is more topologically relaxed than the conformation of xrRNA1 and xrRNA3 and thus likely to be more prone to digestion by XRN1. This explains why PaRV xrRNA2 has poor XRN1 resistance and produces only a fractional amount of sfRNA-2 (Fig 1A, C). To further validate the identified xrRNAs, we assessed the effect of PK disruption on the generation of PaRV sfRNAs in vitro. Mutations that disrupt base pairing in PKs of xrRNA1, xrRNA2 and xrRNA3 completely abolished XRN1 resistance in the corresponding regions of PaRV 3'UTR and prevented the generation of sfRNA-1, sfRNA-2, and sfRNA-3, respectively (Fig 3C). This confirms that PK-forming stemloops SLI-III represents structural determinants of sfRNA biogenesis in PaRV 3'UTR. Finally, we queried the origin of the multiple xrRNAs in PaRV. The structure-based sequence alignment revealed a high level of homology between PaRV xrRNAs, with xrRNA-2 being the most divergent (Fig 3D). This indicates that multiple copies of PaRV SLs likely appeared due to duplication events with subsequent accumulation of mutations in xrRNA-2, which decreased XRN1 resistance.

SHAPE-guided folding of PCV 3'UTR identified three copies of the structural element, each containing PK-forming SLs and two small SLs, followed by two short hairpins at 3'SL (Fig 3E). The PK-forming SLs of PCV had the features of class $1 \mathrm{~b}$ xrRNAs, such as 3nt P1 stem, 3nt long range PK formed by L2 loop and an internal PK formed by nucleotides CGG/A located between P3 and P1 (Fig 3E, F). In addition, SLII and SLIII of PCV contained a noncanonical $A-C$ pairing in the base of the $\mathrm{P} 1$ stem, which is a distinctive feature of class 1b xrRNAs. The SLI did not involve noncanonical base pairing and had a long P2 helix, which hasn't been previously observed in flavivirus xrRNAs (Fig 3E, F). Each of the identified SLs mapped accurately to the 5'-ends of PCV sfRNAs (Supplementary Figure 2). Together with the structural features of SLs, this allowed us to identify them as putative xrRNAs. Their XRN1 
resistance was further confirmed by $\mathrm{XRN1}$ digestion assay, which showed that mutations in L2 loop of SLI, SLII and SLIII preventing the formation of PK interactions abolished production of sfRNA-1, 2 and 3, respectively (Fig 3G). Structure and sequence alignment demonstrated that despite the apparent structural difference of SLI, all three xrRNAs of PCV had a high degree of homology in all their structural elements except for the P2 helix and thus likely resulted from a duplication of a single ancestral structure (Fig $3 \mathrm{H})$.

In conclusion, we for the first time, determined secondary structures of the 3'UTRs of cISFs. We demonstrated that 3'UTRs of PaRV and PCV contain multiple copies of class 1b xrRNAs and a conserved 3'SL. In addition, PaRV was found to contain additional xrRNA-like elements that could represent xrRNAs that lost their functions due to the accumulation of mutations. PARV 3'UTR also has xrRNA that lost its XRN1 resistance partially (xrRNA-2). We also obtained the evidence that multiple xrRNAs in the 3'UTRs of PaRV and PCV emerged due to duplication, which happened early enough in their evolution to allow accumulation of sequence difference between xrRNAs of each virus while maintaining structural conservancy.

\section{Anopheles-associated cISFs contains a single copy of xrRNA, which exhibits characteristics of class $1 \mathrm{a}$ and class $1 \mathrm{~b}$ xrRNAs}

Recently discovered Anopheles-associated cISFs are the most phylogenetically divergent group of insect-specific flaviviruses that were suggested to represent the closest clade to the common ancestors of ISFs and MBFs ${ }^{34}$. In this study, we used KRBV to represent this clade, which appeared to be the only flavivirus analysed so far that produced only one sfRNA species (Fig 1A, C). SHAPE analysis of KRBV 3'UTR demonstrated that it does not contain a typical 3'SL element, which was believed to be conserved in all flaviviruses (Fig 4A). Instead, it has a 3'-terminal stretch of unstructured RNA preceded by a large stem-loop 
with a three-way junction (Fig 4A), which structurally resembles xrRNAs of TBFs and NKVFs ${ }^{19}$. In addition, SHAPE indicated that nucleotides in the terminal loop of this structure are likely paired, suggesting a potential PK between U429-A432 and U476-A480 (Fig 4A). However, we cannot confidently say whether this PK exists as the U476-A480 region was located within the SHAPE primer and did not produce structural data. In any case, this structure was unlikely to be responsible for the biogenesis of KRBV sfRNA as the location of this SL did not match the observed sfRNA length (Fig 1A, C).

Another structured region was identified at the 5' end of KRBV 3'UTR. It contained a stem-loop with a 3-way junction, a long-range and an internal pseudoknot followed by a simple stem-loop element (Fig 4A). Given the location of this structure at the position that matched the observed length of KRBV xrRNA and the organisation of this element, we assumed that this was a putative xrRNA. Although this SL had features of class $1 \mathrm{~b}$ xrRNA, such as 3nt P1 stem and no unpaired nucleotide between P2 and P3, it lacked noncanonical A-C pairing at the base of $P 1$ stem and had a 5nt-PK (Fig 4A, B). This length of PK is typical for class 1a xrRNAs, while all class $1 \mathrm{~b}$ xrRNAs characterised to date contain a very conserved 3nt $\mathrm{PK}^{26}$. In addition, it contained a very long P2 helix (Fig 4A, B) similar to xrRNA-1 of PCV (Fig 3F, G), which does not occur in other members of the Flavivirus genus. The mutation that prevents the formation of 5nt long range PK in KRBV SLI was found to abolish XRN1 resistance and resulted in nearly complete degradation of XRN1-treated RNA (Fig 4C). This indicates that the identified structural element is a key determinant of XRN1 resistance and sfRNA biogenesis in KRBV.

To determine if the identified organisation of 3'UTR and structure of xrRNA are conserved within Anopheles associated flaviviruses (AnFV1, AnFV2), we first performed sequence alignment (Supplementary figure 4A) of the entire 3'UTRs of KRBV and AnFV1 (AnFV1 and AnFV2 have identical 3'UTRs). The alignment revealed a high degree of 
homology at the 3'-end of their 3'UTRs. Subsequent computational folding of the homologous region of AnFV1 demonstrated the same complex stem-loop identified in KRBV (Supplementary figure 4B). At the 3'-end of AnFV1 3'UTR the alignment identified the region with an almost identical sequence to KRBV xrRNA, which contained a large gap (Fig 4D, Supplementary figure $4 \mathrm{~A})$. The in silico folding of this region revealed the structure that resembles KRBV xrRNA, including 5nt PK while having a short P2 helix (Fig 4E). Based on these results, we concluded that Anopheles-associated ISFs have a specific 3'UTR topology, which is conserved within the group, but distinct from other flaviviruses.

In conclusion, we for the first time, determined the structure of the 3'UTR of Anophelesassociated cISF. We found that it contains two conserved structural elements- one is xrRNA, and another one is TBF-like SL. The lack of xrRNA duplications in this group of viruses suggests that they are likely positioned phylogenetically prior to the evolutional event, resulting in structure duplication and are potentially ancestral to all other ISFs. In addition, the fact that xrRNAs of Anopheles-associated flaviviruses share characteristics of cISF class $1 \mathrm{~b}$ and MBF/dISF class 1a xrRNAs indicates that they may resemble the ancestral form of xrRNA, from which both subclasses of xrRNAs emerged later in the evolution.

\section{xrRNAs of ISFs and MBFs evolved from structures similar to xrRNAs of Anopheles- associated ISFs and were independently duplicated in each clade}

To examine phylogenetic relationships between XRN1-resistant elements of different ISFs, we performed structure-based alignments of identified and predicted xrRNAs. Considering the potential origin of dISFs from MBFs, MBF xrRNAs were aligned with dISF xrRNAs, while cISF xrRNAs were aligned separately. Alignment demonstrated that dISF xrRNAs diverged from MBF xrRNAs in two independent evolutional events (Fig 5A). In addition, it showed that some MBF xrRNAs (e.g. DENV1 and DENV3 xrRNA-2) had higher 
similarity with dISF xrRNAs than with another xrRNA of the same virus (Fig 5A). Given that dISFs are phylogenetically basal to the DENV group (Supplementary Figure 5), this indicates that duplication of xrRNAs in MBFs likely occurred when the DENV clade diverged. This is due to the evidence that the most basal member of the DENV group, DENV4, does not contain duplicated $x \mathrm{RRNA}^{27}$. MBFs generally have a higher similarity between corresponding xrRNAs of different viruses than between individual xrRNAs within the same virus. However, cISFs do not follow this trend and commonly exhibit higher homology between different xrRNAs of the same virus than between xrRNAs of different viruses (Fig 5B). This indicates that duplications of xrRNAs in ISFs were more frequent, while xrRNAs of MBFs likely evolved after a single duplication event. In addition, structure-based alignment identified xrRNAs of Anopheles-associated ISFs that lack structure duplication as the most basal and likely the most ancestral to all other cISFs (Fig 5B).

To further elucidate evolutional relationships between 3'UTR topology of ISFs and MBFs we performed structure informed sequence alignment of the complete 3'UTRs of all cISFs, dISFs and MBFs and performed maximum-likelihood phylogenetic inferences. We also built covariance models for extended xrRNAs (canonical xrRNA followed by small SL) of MBF, dISF, cISF and Anopheles-associated ISFs to identify their consensus structures. The 3'UTR alignment demonstrated the ancestral position of the Anopheles viruses to both - cISF and dISF clades (Fig 5C, Supplementary figure 5). The topology of the 3'UTR phylogenetic tree was congruent with the overall topology of the viral polyprotein phylogenetic tree (Supplementary figure 5). This indicates the co-evolution of both the polyprotein and 3'UTR sequences. The clade of clSFs containing two copies of xrRNAs (CFAV, KRV and Aedes flavivirus [AEFV]) is more closely related to Anopheles-associated ISFs. In contrast, MBFs containing multiple copies of xrRNAs have a far greater evolutionary distance (Fig 5C, Supplementary Figure 5). This further confirms that the evolution of clSFs involved two 
separate events of xrRNA duplications. In the MBF/dISF part of the tree, the Yellow fever group was the closest to the Anopheles-associated ISF-like ancestor, with both containing single xrRNA. It was followed by two branches of dISFs all containing single xrRNA and then the rest of MBFs with two xrRNA structures. Therefore the emergence of MBF clade involved a single xrRNA duplication event. The structural alignment of individual xrRNAs (Fig 5A and B) indicates that MBFs and cISFs did not evolve from the common ancestor with duplicated structures but rather acquired duplications due to independent evolutionary events (Fig 5C). This also shows that dISFs did not lose extra copies of xrRNA while diverging from MBF and transitioning from dual-host to single-host life cycle as previously suggested. Rather, they branched off before the duplication event in the MBF clade occurred (Fig 5C). Moreover, it appears that another duplication event occurred in the dISF clade, which resulted in two copies of xrRNAs being present in BinJV 3'UTR.

The results of phylogenetic analysis collectively indicate that clSFs and MBFs diverged from a common ancestor similar to Anopheles-associated ISFs. The evolution of ISFs indicates a strong selective pressure for producing additional XRN1 resistant elements either via duplication of existing xrRNAs (MBFs, clSFs) or by gaining additional XRN1 resistance through smaller novel xrRNA structures (dISFs). The fact that duplication of xrRNAs independently occurred several times in flavivirus evolution and was further selected indicates that multiple sfRNA species confer an advantage in viral fitness irrespective of which hosts they replicate.

\section{ISFs can tolerate the loss of individual sfRNAs, but not a complete sfRNA deficiency}

A previous study on Dengue viruses proposed that different xrRNAs within an individual 3'UTR produced functionally divergent sfRNA species ${ }^{13}$. One of these sfRNAs (shorter) was beneficial for virus replication in the insect host, and another sfRNA (longer) for 
replication in vertebrates ${ }^{13}$. Switching between the production of longer and shorter sfRNAs was suggested to occur as part of adaptation for virus shuttling between mosquitoes and vertebrates ${ }^{13,27}$. Here we demonstrated that single host flaviviruses also contain duplicated xrRNAs and produce multiple sfRNA species. As ISFs don't switch hosts, the duplications of xrRNAs in these clades should have a different biological function. It is also unclear whether different sfRNA species produced by ISFs are functionally redundant or divergent.

To clarify this matter, we generated mutant ISFs deficient in the production of sfRNAs and assessed their replication. If sfRNA isoforms are redundant, we expected that viruses would tolerate deficiency in single sfRNA but become attenuated when production of all sfRNAs is impaired. If individual sfRNA species have unique, specialised functions, we expected that loss of each sfRNA should equally impair virus replication. Therefore, we introduced mutations that prevent PK formation by SLI, SLII and SLIII of PaRV and PCV generating mutants in PK1', PK2' and PK3', respectively. We also generated PK1'2'3' mutants for PaRV and PCV in which all three PKs were mutated. As BinJV contains only two canonical xrRNAs we generated PK1', PK2' and PK1'2' mutants for this virus.

Northern blot analysis of RNA isolated at 7dpi demonstrated that PK1', PK2' and PK3' mutations abolished production of sfRNA-1, sfRNA-2 and sfRNA-3, respectively, by PaRV and PCV (Fig 6A). For PaRV, PK3' mutation also reduced sfRNA1 and sfRNA2, suggesting some long range interactions between xrRNAs. As expected, triple mutant PK1'2'3' of PARV was completely deficient in the production of all three sfRNAs (Fig 6A). However, two attempts to recover PK1'2'3' mutant PCV yielded very low viral titers $\left(\sim 10^{3}\right.$ $\mathrm{FFU} / \mathrm{mL}$ vs $\sim 10^{6-7} \mathrm{FFU} / \mathrm{mL}$ observed for other mutants and WT virus). Moreover, the recovered virus accumulated mutations in xrRNA1 and xrRNA2 that restored the production of sfRNA-1 and sfRNA-2, respectively (Fig 6A). This indicates that complete sfRNA deficiency is detrimental for PCV replication and reveals a strong selective pressure to restore sfRNA 
production. In the case of BinJV, PK1' and PK2' mutants had decreased but not completely abolished production of corresponding sfRNAs, consistent with the in vitro assay results (Fig 2D) and explained by the additional XRN1 resistance present in the novel xrRNAs (Fig 2D, E). Accordingly, the PK1'2' mutant of BinJV showed reduced but not completely abolished production of both sfRNA-1 and sfRNA-2.

To determine how complete and partial deficiency in sfRNA affects replication of ISFs, we compared growth kinetics of generated mutants and corresponding WT viruses in RNAi-competent mosquito cells RML-12. In PaRV, for which we obtained a complete set of sfRNA-deficient phenotypes, the lack of sfRNA-2 (PK2' mutant) did not affect virus replication (Fig 6B). However, this can be attributed to the overall low abundance of this sfRNA even in the WT virus which is caused by the unusual fold of xrRNA2 (Figs 1A, 1C, 6A). PaRV mutants deficient in sfRNA-1 (PK1' mutant), sfRNA1 and sfRNA-3 (PK3' mutant - no sfRNA3 and much less sfRNA1), and all three sfRNAs (PK1'2'3' mutant) were attenuated at 3 and 5dpi (Fig 6B). However, by 7dpi, PK1' and PK3' mutants overcame the initial attenuation and reached replication levels comparable to the WT virus, while replication of PK1'2'3' mutant remained significantly impaired (Fig $6 \mathrm{~B}$ ). This indicates that the loss of individual sfRNAs could be tolerated. In contrast, the loss of all three sfRNAs substantially reduced virus replication through the entire course of infection. Notably, individual PK mutants of PCV replicated at the same levels as WT virus (Fig 6B), which further indicates that deficiency in individual sfRNAs is tolerable for ISFs and suggests that their functions are likely to be redundant. Moreover, the recovery of sfRNA1 and sfRNA2 production by PK1'2'3' mutant of PCV due to reversions restored replication of this virus, which initially had a very low titer. PK1', PK2' and PK1'2' mutants of BinJV also replicated at the levels comparable to the WT virus at all time points (Fig 6B). However, these mutants were not fully deficient in either sfRNA-1 or sfRNA-2 due to the involvement of novel xrRNAs that still enabled the production 
of corresponding sfRNAs (albeit at reduced levels) despite the mutations in xrRNA1 and xrRNA2 (Fig 6A).

Collectively, our results indicate that sfRNA species of different length are likely to be functionally redundant in ISFs. Therefore, we believe that duplication of xrRNAs ensures sfRNA production even if one of the structures becomes not resistant to XRN1 digestion due to inadvertent mutations. In addition, duplicated xrRNAs enable the production of smaller sfRNA even when the upstream xrRNA fails to provide XRN1 resistance due to misfolding or conformational transition into a non-resistant state. We also showed that in dISF a similar backup mechanism for sfRNA production is provided by novel xrRNAs. The presence of these novel xrRNAs, which is unique for dISFs, explains why most dISFs tolerated the loss of duplicated xrRNAs without compromising the robustness of sfRNA production and virus viability.

\section{Discussion}

Herein we demonstrated that cISFs and dISFs produce sfRNAs by employing the XRN1-resistance mechanism. Using RNA SHAPE we, for the first time, determined secondary structures of complete 3'UTRs of the divergent representatives of cISF and dISF clades and experimentally identified structured RNA elements that enable XRN1 resistance and production of sfRNAs in these viruses. We also confirmed XRN1 resistance of the identified xrRNAs by in vitro XRN1 digestion assays and analysis of the xrRNA mutant viruses. We then used structure-guided phylogenetic analysis of flavivirus xrRNAs and 3'UTRs to understand the role of XRN1 resistance and SfRNA production in the evolution of flaviviruses.

All dual host flaviviruses tested to date were shown to produce sfRNA by employing XRN1-resistant structured RNA elements ${ }^{7}$. In addition, the production of sfRNA was 
demonstrated for cISF CFAV ${ }^{19}$. In this study, we showed that SfRNA is produced by a wide variety of flaviviruses, including cISFs and dISFs. Conservation of sfRNA production in the highly divergent ISFs, dISF BinJV and Anopheles-associated cISF KRBV indicates the crucial importance of sfRNA for flavivirus replication in insect hosts. Moreover, considering that Anopheles-associated ISFs are believed to be the most closely related viruses to the common flaviviral ancestor ${ }^{34}$, the production of sfRNA by KRBV indicates that the ability to resist RNA degradation by $\mathrm{XRN1}$ and generate sfRNAs was fixed very early in flavivirus evolution. This further emphasises the importance of sfRNA for flaviviruses.

Most mosquito-borne vertebrate infecting flaviviruses are known to produce at least two sfRNA species due to the presence of duplicated xrRNAs in their 3'UTRs $^{7}$, while ISFs have been predicted to lack these duplications ${ }^{27,30}$. In addition, a previous study demonstrated that adaptation of DENV to insect and vertebrate hosts involves switching between the production of shorter and longer sfRNA species, respectively, due to the accumulation of point mutations in corresponding $\operatorname{xrRNAs}^{13,38}$. Longer sfRNA species were found to benefit DENV replication in vertebrates, while shorter sfRNAs were shown to function in mosquito cells ${ }^{13}$. It was, therefore, suggested that duplication of xrRNAs occurred in dual host infecting flaviviruses to enable switching between the production of functionally distinct sfRNAs while adapting to replication in different hosts. Lack of 3'UTR structure duplications in ISFs was proposed as a reason for their host restriction.

Contrary to this, we found that all ISFs analysed in our study except KRBV contained duplicated xrRNAs and produced multiple sfRNAs, although none of the viruses could infect vertebrate cells. Moreover, representatives of the YFV-group ${ }^{24}$ and DENV4 ${ }^{39}$ contain only one $x r R N A$ while replicating in both insect and vertebrate hosts. Collectively, published data and our results herein indicate that duplication of xrRNAs is not a requirement for dual host life cycle and lack of these duplications is not the mechanism of host restriction in ISFs. 
Furthermore, we found that deficiency in the production of individual sfRNAs can be tolerated by ISFs, while lack of all sfRNAs is detrimental for ISF replication fitness. This result is consistent with previous observations by us and others in MBFs. In particular, ZIKV and WNV mutants deficient in single sfRNAs replicated in mosquito and vertebrate cells at the levels comparable to WT virus, whereas mutations that abolish production of both sfRNAs either resulted in a substantial attenuation of the viruses or were lethal ${ }^{10,11,18,40,41}$. Therefore, we concluded that in many flaviviruses, including ISFs, different sfRNA species are functionally redundant and host specialisation of sfRNAs observed in DENV ${ }^{13,38}$ is likely unique to the DENV group.

Another important novel outcome of this study is the discovery of the ancestral form of xrRNA in the 3'UTR of Anopheles-associated cISF KRBV. Together with the structural data for the 3'UTR of KRBV and other ISFs generated here, it enabled us to reconstruct the most complete to date structure-based phylogenetic trees of flavivirus xrRNAs and 3'UTRs and mapping of xrRNA duplication events on this tree. The Anopheles-associated ISFs contains a single copy of the XRN1-resistant element, which has a general topology of class $1 \mathrm{~b}$ xrRNA (that occur in TABV and cISFs) while lacking noncanonical C-A pairing and forming 5-nt pseudoknot, which only occurs in class 1a xrRNAs of MBFs and dISFs. This structure was found to be ancestral for both cISFs and MBFs/dISFs xrRNAs and likely has the same organisation as xrRNA of a common flavivirus ancestor. Notably, 3'UTRs of YFV group are the most closely related to Anopheles-associate viruses on the MBF branch of the phylogenetic tree. They were followed by two independent branches of dISFs and then the rest of MBFs (Fig 5C). Considering that viruses from YFV-group do not contain xrRNA duplications, this indicates that dISFs diverged before the duplication event in the MBF clade and an independent xrRNA structure duplication occurred later in evolution in the BinJV. On the cISF branch, all viruses were found to contain duplicated xrRNAs, which indicates that 
structure duplications occurred very early in the evolution of ISFs. Based on this analysis, we can conclude that duplicated xrRNAs in the 3'UTRs of phylogenetically distant flaviviruses were not the result of divergence from a common ancestor. Instead, they appeared due to convergent evolution with independent duplication events in cISF, MBF and dISF clades. Furthermore, the viruses that contain duplications were strongly selected for in both cISF and MBF lineages, indicating that the presence of multiple xrRNAs has a strong adaptive advantage for both insect-specific and dual host virus life cycles.

Given the functional redundancy of sfRNA species produced from duplicated xrRNAs in ISFs, we propose two potential mechanisms of how this redundancy could benefit viral replication. The robust production of sfRNA is crucial for the efficient replication of flaviviruses. However, individual xrRNAs are dynamic structures that can transition between XRN1-resistant to XRN1 sensitive conformation ${ }^{42}$. Therefore, the most apparent benefit from xrRNA duplication is that downstream copies of xrRNA ensure that sfRNA is produced when upstream xrRNA is not folded into its resistant state. The fact that shorter sfRNAs are always observed in infected cells along with longer sfRNAs indicates that the most upstream xrRNA does not often confer complete XRN1 resistance, thus allowing XRN1 to progress to the subsequent xrRNA, generating a shorter sfRNA variant. Hence, duplications of xrRNAs enable fail-safe sfRNA production despite the thermodynamic fluctuations in individual xrRNAs. Another evolutional benefit from duplicated xrRNAs is protection from mutations in individual XRN1-resistant elements. This mechanism is supported by structural features identified in the 3'UTR of PaRV. This UTR contains four copies of pseudoknot-forming stemloops (SL-PK) that have homologous sequence and structure. However, only two (SLI-PK1 and SLIII-PK3) confer efficient XRN1 resistance and generate substantial amounts of sfRNAs. At the same time, SLIV has lost its XRN1 resistance completely, while SLII accumulated mutations that rendered it significantly less resistant to XRN1 digestion. Despite 
these mutations, PaRV retained its viability due to the production of sfRNAs from the other two copies of xrRNAs, xrRNA1 and xrRNA3. Based on this data, we concluded that xrRNA duplications improve viral fitness by providing a backup strategy for sfRNA production.

Despite the clear benefit of more robust sfRNA production due to structure duplications in the 3'UTR, there are two groups of flaviviruses (YFV group and majority of dISFs) that are evolutionary successful while lacking additional copies of canonical xrRNAs. Our results indicate that dISFs evolved a different strategy to backup sfRNA production. While all dISFs, except BinJV, don't have additional copies of canonical xrRNA, we found that they all contain a novel class of xrRNA, n-xrRNAs, which evolved into XRN1-resistant structures from conserved stem-loop elements CS3/RCS3. Found initially in BinJV and other dISFs, these stem loops acquired the ability to form pseudoknots and confer XRN1 resistance. Our mutational analysis in the live BinJV demonstrated that XRN1 resistance of $n-x r R N A s$ ensures the production of sfRNAs in amounts sufficient to prevent virus attenuation when canonical xrRNAs are mutated. Novel xrRNAs of dISFs represent the simplest organisation of xrRNAs found in flaviviruses so far and demonstrate how very different secondary structures can be used to achieve XRN1 resistant folding. Their discovery explains the mechanism of sfRNA biogenesis in dISFs and expands our knowledge about the structural diversity of xrRNAs in the Flavivirus genus.

The discovery of novel xrRNAs in dISFs and independent xrRNA duplication events in all flavivirus clades shows that flaviviruses evolved multiple strategies to ensure reliable production of sfRNA. Together with highly attenuated phenotypes of viruses completely deficient in sfRNA production, this further highlights the critical importance of sfRNAs for replication of all flaviviruses, including ISFs. Identifying the exact functions of sfRNAs in ISFhost interactions is the next step to understand their functional significance. By discovering the structural determinants of sfRNA biogenesis in diverse ISFs and generating ISF mutants 
deficient in sfRNA production. This study creates a fundamental basis for further explorations of the biological effects of ISF sfRNAs in the loss of function model systems. Such systems were previously employed by us and others to identify molecular functions of MBF sfRNAs ${ }^{10-}$ 12,18 and, through this study, are now also available for ISFs.

\section{Materials and methods}

\section{Cell Culture}

Aedes albopictus larvae cells C6/36 (ATCC - CRL-1660) and Aedes aegypti larvae cells Aag2 (ATCC - CCL-125) were obtained from the ATCC. Aedes albopictus larvae cells RML-12 $2^{43}$ were a gift from Prof. Robert Tesh (UTMB, USA). C6/36 were cultured in Roswell Park Memorial Institute 1640 medium (RPMI 1640). Aag2 cells were cultured in 1:1 mixture of Schneider's Drosophila medium and Mitsuhashi \& Maramorosch medium (Sigma, USA). RML-12 cells were cultured in Leibovitz's L-15 medium supplemented with $10 \%$ tryptosephosphate broth. All culture media were supplemented with 10\% Fetal Calf Serum (FCS), $100 \mu \mathrm{g} / \mathrm{mL}$ streptomycin, $100 \mathrm{U} / \mathrm{mL}$ penicillin, and $2 \mathrm{mM} \mathrm{L-glutamine.} \mathrm{Cells} \mathrm{cultured} \mathrm{at} 28^{\circ} \mathrm{C}$ with $5 \% \mathrm{CO}_{2}$ or in sealed containers. All cell culture media and reagents were from Gibco, USA, unless otherwise specified.

\section{Processing of mosquitoes}

All of the mosquito samples tested in this study were archival and collected for previous study ${ }^{34}$. Mosquitoes were snap-frozen in liquid nitrogen and shipped for processing on dry ice. Total RNA was then isolated from individual mosquitoes and used for RT-PCR screening with Karumba virus (KRBV) genome primers KRBV-F and KRBV-R (Supplementary Table 2). RT-PCR was performed using SSIII One-Step RT-PCR Kit with Platinum Taq (Invitrogen, USA) according to the manufacturer's recommendations. Each RTPCR reaction contained $1 \mu \mathrm{g}$ of mosquito RNA. The cycling conditions were $15 \mathrm{~min}$ at $60{ }^{\circ} \mathrm{C}$, 2 min at $95^{\circ} \mathrm{C} ; 40$ cycles of $15 \mathrm{~s}$ at $95{ }^{\circ} \mathrm{C}, 30 \mathrm{~s}$ at $51^{\circ} \mathrm{C}, 35 \mathrm{~s}$ at $68^{\circ} \mathrm{C}$; and a final extension 
for $5 \mathrm{~min}$ at $68^{\circ} \mathrm{C}$. PCR products were then separated in $2 \%$ agarose gel, and DNA was extracted from the gel using MinElute Gel Extraction Kit (Qiagen, Germany). Purified DNA was Sanger sequenced and matched to KRBV RefSeq sequence NC_035118.1. RNA samples confirmed to contain KRBV were used for further analyses.

\section{Viruses and infection}

Binjari (BinJV), Parramatta River (PaRV), Palm Creek (PCV) and Hidden Valley (HVV) viruses were previously isolated from Australian mosquito populations ${ }^{2,31-33}$ and passaged in C6/C6 cells for a total of 3-6 passages. For the generation of the virus stocks used in the study $\mathrm{C} 6 / 36$ cells were infected at $\mathrm{MOI}=0.1$, and culture fluids were harvested at $7 \mathrm{dpi}$. Culture fluids were then cleared from cell debris by centrifugation at $3000 \mathrm{x} \mathrm{g}$ for $15 \mathrm{~min}$ at $4^{\circ} \mathrm{C}$ and stored at $-80{ }^{\circ} \mathrm{C}$ in single-use aliquots. Virus titers were determined by foci-forming immunoassay on $\mathrm{C} 6 / 36$ cells. All infections were performed at the indicated Multiplicity of Infection (MOI) by incubating cells with $50 \mu \mathrm{L}$ of inoculum per $\mathrm{cm}^{2}$ of growth area for $1 \mathrm{~h}$ at $28^{\circ} \mathrm{C}$. Inoculated cells were then maintained in the growth medium containing a reduced amount of FCS (2\%) to prevent overgrowth.

\section{Foci-forming immunoassay}

Ten-fold serial dilutions of culture fluids were prepared in RPMI media supplemented with $2 \% \mathrm{FCS}$, and $25 \mu \mathrm{l}$ of each dilution were used to infect $10^{5} \mathrm{C} 6 / 36$ cells grown in 96 -well plates. After $2 \mathrm{~h}$ incubation with the inoculum, $180 \mu \mathrm{l}$ overlay media was added to the cells and incubated at $27{ }^{\circ} \mathrm{C}$ in $5 \% \mathrm{CO}_{2}$. The overlay media contained one part $\mathrm{X} \mathrm{M} 199$ medium (containing 5\% FCS, $100 \mu \mathrm{g} / \mathrm{mL}$ streptomycin, $100 \mathrm{U} / \mathrm{mL}$ penicillin, and $2.2 \mathrm{~g} / \mathrm{L} \mathrm{NaHCO}$ ) and another part $2 \%$ carboxymethyl cellulose (Sigma-Aldrich, USA). At 3 days post-infection, cells were fixed with $100 \mu \mathrm{L} /$ well of $80 \%$ acetone for 20 min at $-20^{\circ} \mathrm{C}$, washed with PBS, thoroughly dried and blocked for 30 min with $150 \mu \mathrm{L} /$ well of ClearMilk blocking solution (Pierce, USA). Cells were then incubated with $50 \mu \mathrm{L} /$ well of mouse monoclonal antibody to flavivirus envelope protein (4G2 for detection of BinJV² and HVV 33 , 7D11 for PaRV44 and 5G12 for 
$\mathrm{PCV}^{44}$ ), diluted in 1:100 for $1 \mathrm{~h}$, followed by $1 \mathrm{~h}$ incubation with $50 \mu \mathrm{l} /$ well of $1: 800$ dilution of goat anti-mouse IRDye $800 \mathrm{CW}$ secondary antibody (LI-COR, USA). All antibodies were diluted with Clear Milk blocking buffer (Pierce, USA), and incubations were performed at 37 ${ }^{\circ} \mathrm{C}$ for $1 \mathrm{~h}$. After each incubation with antibody, plates were washed 5 times with phosphate buffered saline (PBS) containing 0.05\% Tween 20 (PBST). Plates were then scanned using an Odyssey CLx Imaging System (LI-COR) (42 $\mu \mathrm{m}$; medium; $3.0 \mathrm{~mm}$ ). Virus replication foci were counted using the Image Studio Lite software (v5.2.5, LI-COR, USA), and titres were determined based on dilution factors and expressed as focus forming units per $\mathrm{mL}\left(\mathrm{FFU} \mathrm{mL}^{\wedge}\right.$ 1).

\section{RNA isolation}

Total RNA was isolated from cells and mosquitoes using TRIreagent (Sigma, USA). Individual mosquitoes were homogenised in $500 \mu \mathrm{L}$ TRIreagent for $5 \mathrm{~min}$ at $30 \mathrm{~Hz}$ using a Tissue Lyser II (Qiagen, USA) before RNA isolation. Viral RNA from cell culture fluids was isolated using QIAamp Viral RNA Mini Kit (Qiagen, USA). All RNA isolation procedures were conducted according to the manufacturer's instructions. RNA concentrations were determined on NanoDrop One microspectrophotometer (ThermoFisher Scientific, USA), and RNA purity was assessed by $\mathrm{OD}_{260} / \mathrm{OD}_{280}$ and $\mathrm{OD}_{260} / \mathrm{OD}_{230}$ ratios.

\section{Northern blotting}

Total RNA (2.5-10 $\mathrm{\mu g}$ ) was mixed with an equal volume of Loading Buffer II (Ambion, USA), denatured at $85^{\circ} \mathrm{C}$ for $5 \mathrm{~min}$ and chilled on ice for $2 \mathrm{~min}$. Samples were then subjected to electrophoresis in 6\% polyacrylamide TBE-Urea gels (Invitrogen, USA). Electrophoresis was performed for 90 min in 1x Tris-Borate-EDTA buffer pH8.0 (TBE). Gels were stained with ethidium bromide to visualise rRNA and documented using Omnidoc imager (Cleaver Scientific, UK). RNA was then electroblotted onto Amersham Hybond-N ${ }^{+}$nylon membrane (GE Healthcare, USA) for $90 \mathrm{~min}$ at $35 \mathrm{~V}$ in $0.5 \mathrm{x}$ TBE using the TransBlot Mini transfer apparatus (Bio-Rad, USA) and UV-crosslinking at $1200 \mathrm{kDj} / \mathrm{cm}^{2}$. Membranes were pre- 
hybridised at $50^{\circ} \mathrm{C}$ in ExpressHyb Hybridization Solution (Clontech, USA) for $1 \mathrm{~h}$. The probes were prepared by end labelling 10 pmoles of DNA oligonucleotide complementary to the sfRNA (Supplementary Table 2) with [y-32P]-ATP (Perkin-Elmer, USA) using T4 polynucleotide kinase (NEB, USA) and purified from unincorporated nucleotides by gel filtration on Illustra MicroSpin G-25 Columns (GE Healthcare, USA). Hybridisation was performed overnight at $50{ }^{\circ} \mathrm{C}$ in ExpressHyb Hybridisation Solution (Clontech, USA). After hybridisation, membranes were rinsed, washed 4 × 15 min with Northern Wash Buffer (1\% sodium dodecyl sulphate [SDS], $1 \%$ saline-sodium citrate [SSC]) at $50^{\circ} \mathrm{C}$ and exposed to a phosphor screen (GE Healthcare, USA) overnight. Signal detection was performed on Typhoon FLA 7000 Imager (GE Healthcare, USA).

\section{RNAi knock-down}

The dsRNA against Ae. aegypti XRN1/pacman was generated by in vitro transcription (IVT). The DNA templates for IVT were prepared by amplifying a $\sim 3 k b$ fragment of XRN1/pacman mRNA with primers designed to incorporate T7 promoter into PCRproducts in either forward or reverse directions (Supplementary Table 2) to generate templates for the synthesis of sense and antisense RNA strands. Total RNA $(1 \mu \mathrm{g})$ isolated from Aag2 cells was used as a template for RT-PCR performed using SSIII One-Step RTPCR Kit with Platinum Taq (Invitrogen, USA) according to the manufacturer's recommendations. The cycling conditions were $15 \mathrm{~min}$ at $60{ }^{\circ} \mathrm{C}, 5 \mathrm{~min}$ at $94{ }^{\circ} \mathrm{C} ; 5$ cycles of $15 \mathrm{~s}$ at $95^{\circ} \mathrm{C}, 30 \mathrm{~s}$ at $50{ }^{\circ} \mathrm{C}, 3 \mathrm{~min}$ at $68^{\circ} \mathrm{C} ; 35$ cycles of $15 \mathrm{~s}$ at $95^{\circ} \mathrm{C}, 30 \mathrm{~s}$ at $60{ }^{\circ} \mathrm{C}, 3 \mathrm{~min}$ at $68{ }^{\circ} \mathrm{C}$ and a final extension for $5 \mathrm{~min}$ at $68^{\circ} \mathrm{C}$. PCR products were then separated in $1 \%$ agarose gel, and DNA was extracted from the gel using Monarch Gel Extraction Kit (NEB, USA). In vitro transcription was then performed using $1 \mu \mathrm{g}$ of purified DNA templates and MEGAscript T7 Transcription Kit (Invitrogen, USA). RNA was purified by LiCl precipitation, $50 \mu \mathrm{g}$ of each ssRNA were combined in a total volume of $100 \mu \mathrm{L}$ and denatured by heating at $85^{\circ} \mathrm{C}$ for 10 min followed by gradual cooling to RT to produce dsRNA. The RNA annealing 
was validated by electrophoresis in $1 \%$ native agarose gel. Aag2 cells were then transfected with $2 \mu \mathrm{g}$ of the resulted dsRNA per $10^{6}$ cells using Lipofectamine 2000 (Invitrogen, USA) following transfection in suspension protocol ${ }^{45}$. Cells were plated into 6 -well plates and at 24 h post transfection infected with PaRV, PCV or BinJV at MOI=1. At 2 dpi cells were lysed in TRIreagent (Sigma, USA), and total RNA was isolated.

\section{3'UTR cloning}

Total RNA was isolated from C6/36 cells infected with PaRV, PCV, BinJV or HVV at 5dpi and from KRBV-positive mosquitoes, and RT-PCR was used to amplify the fragment of the viral genome containing 3'UTR and the small terminal part of NS5 gene. RT-PCR was performed using SSIII One-Step RT-PCR Kit with Platinum Taq (Invitrogen, USA) according to the manufacturer's recommendations. PCR primers (Supplementary Table 2) were 5'phosphorylated, and forward primers were designed to incorporate T7 promoter at the 5'-end of the amplicons. The cycling conditions were $15 \mathrm{~min}$ at $60^{\circ} \mathrm{C}, 2 \mathrm{~min}$ at $95^{\circ} \mathrm{C} ; 35$ cycles of $15 \mathrm{~s}$ at $95^{\circ} \mathrm{C}, 30 \mathrm{~s}$ at $55^{\circ} \mathrm{C}, 40 \mathrm{sec}$ at $68^{\circ} \mathrm{C}$; and a final extension for $5 \mathrm{~min}$ at $68^{\circ} \mathrm{C}$. PCR products were then separated in $2 \%$ agarose gel, purified using Monarch Gel Extraction Kit (NEB, USA) and ligated with Smal-digested and dephosphorylated pUC19 vector using Blunt/TA Ligase Mastermix (NEB, USA). Ligation was performed overnight at $16{ }^{\circ} \mathrm{C}$, and products were transformed into NEB5 $\alpha$ chemically competent cells (NEB, USA) and plasmids were isolated using QIAprep Spin Miniprep Kit (Qiagen, Germany).

\section{XRN1 resistance assay}

Plasmids were linearised by restriction digest and purified using Monarch PCR and DNA Clean-up Kit (NEB, USA). 3'UTRs were in vitro transcribed from $1 \mu \mathrm{g}$ of linearised plasmids using MEGAscript T7 Transcription Kit (Invitrogen, USA) according to the manufacturer's recommendations. RNA was purified by $\mathrm{LiCl}$ precipitation and analysed by electrophoresis in a 1.2\% denaturing agarose gel. RNA was then refolded in NEB3 buffer by heating at $85^{\circ} \mathrm{C}$ for $5 \mathrm{~min}$ followed by gradual cooling to $28^{\circ} \mathrm{C}$. The refolded RNA $(1 \mu \mathrm{g})$ was 
incubated with $1 \mathrm{U}$ XRN1 (NEB, USA) and 10U RppH (NEB, USA) in 20 $\mu \mathrm{L}$ of reaction mixture containing 1x NEB3 buffer (NEB, USA) and 1u/ $\mu \mathrm{L}$ RNasin RNase Inhibitor (Promega, USA). Incubation was performed for $2 \mathrm{~h}$ at $28{ }^{\circ} \mathrm{C}$. The reaction was stopped by adding $20 \mu \mathrm{L}$ of Loading Buffer II (Ambion, USA), heating for $5 \mathrm{~min}$ at $85^{\circ} \mathrm{C}$ and placing on ice. The entire volume was then loaded into $6 \%$ polyacrylamide TBE-Urea gels (Invitrogen, USA), and electrophoresis was performed for $90 \mathrm{~min}$ in 1xTBE. Gels were stained with ethidium bromide and documented using an Omnidoc imager (Cleaver Scientific, UK).

\section{Ligation-mediated sequencing of sfRNAs}

Total RNA from infected C6/36 cells $(20 \mu \mathrm{g})$ was denatured by heating at $75^{\circ} \mathrm{C}$ for 5 min, then placed on ice. Denatured RNA was circularised by incubation with 10U T4 RNA Ligase 1 (NEB, USA) in $20 \mu \mathrm{L}$ of the reaction mixture containing $1 \times$ T4 RNA Ligase Buffer (NEB, USA), 1mM ATP (NEB, USA), 12.5\% PEG8000 and 1u/ $\mu \mathrm{L}$ RNasin RNase Inhibitor (Promega, USA). Incubation was performed $\mathrm{O} / \mathrm{N}$ at $16{ }^{\circ} \mathrm{C}$, then $10 \mu \mathrm{L}$ of the mix was used as the template for RT reaction with LigSeq-RT primer (Supplementary Table 2) and SuperScript IV RT enzyme (Invitrogen, USA), which was performed according to the manufacturer's recommendations. RNA was then removed from the mixture by incubation with $5 \mathrm{U}$ RNase $\mathrm{H}$ (NEB, USA) and 10 $\mathrm{\mu g}$ DNase-free RNase A (ThermoFisher Scientific, USA) for 20 min at 37 ${ }^{\circ} \mathrm{C}$. RNA-free cDNA $(5 \mu \mathrm{L})$ was used as a template for PCR with PrimeStar GXL Polymerase (Takara, Japan) and back-to-back PCR primers (LigSeq-F and LigSeq-R, Supplementary Table 2) designed in the proximity to the 3'-end of viral 3'UTRs. The cycling conditions were $1 \mathrm{~min}$ at $98{ }^{\circ} \mathrm{C} ; 40$ cycles of $15 \mathrm{~s}$ at $98^{\circ} \mathrm{C}, 45 \mathrm{sec}$ at $68^{\circ} \mathrm{C}$; and a final extension for $5 \mathrm{~min}$ at $68{ }^{\circ} \mathrm{C}$. Amplicons were separated in $2 \%$ agarose gel, purified using Monarch Gel Extraction Kit, and Sanger sequenced with LigSeq-F and $-\mathrm{R}$ primers. The resulted sequences were aligned to the reference genomes using CLC Main Workbench v8.1.0 (Qiagen, Germany) and nucleotide positions found to be ligated to the known 3'-end of the 3'UTRs were identified as $5^{\prime}$-ends of the sfRNAs. 


\section{Selective 2' Hydroxyl Acylation analyzed by Primer Extension (SHAPE)}

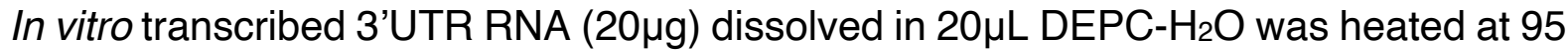
${ }^{\circ} \mathrm{C}$ for $2 \mathrm{~min}$, then placed on ice for $2 \mathrm{~min}$. RNA was then refolded by incubation at $28{ }^{\circ} \mathrm{C}$ for 30 min in $150 \mu \mathrm{L}$ of RNA folding buffer (100 mM HEPES pH 8.0, $100 \mathrm{mM} \mathrm{NaCl,} 6 \mathrm{mM} \mathrm{MgCl}_{2}$, 0.5 U/ $\mu \mathrm{L}$ RNasin PLUS RNase Inhibitor (Promega, USA)). The solution was equally divided between two tubes (72 $\mu \mathrm{L}$ each) and incubated for 30 min at $28{ }^{\circ} \mathrm{C}$ with either $8 \mu \mathrm{L}$ of $50 \mathrm{mM}$ NMIA (ThermoFisher Scientific, USA) in DMSO (+ reaction) or $8 \mu \mathrm{L}$ of DMSO (- reaction). RNA solutions were mixed with $4 \mu \mathrm{L} 3 \mathrm{M} \mathrm{NaOAc} p H$ 5.2, $2 \mu \mathrm{L} 100 \mathrm{mM}$ EDTA, $1 \mu \mathrm{L}$ Glycogen $(20 \mathrm{mg} / \mathrm{mL})$ and $350 \mu \mathrm{L}$ ice-cold $100 \%$ ethanol and incubated overnight at $-80{ }^{\circ} \mathrm{C}$ to precipitate RNA. RNA was pelleted by 30 min of centrifugation at $10,000 \times \mathrm{g}, 4{ }^{\circ} \mathrm{C}$, washed with $75 \%$ DEPC-EtOH and resuspended in 10 $\mathrm{LL}$ 0.5x TE buffer $\mathrm{pH}$ 8.0. Five microliters of each RNA solution were combined with $6 \mu \mathrm{L}$ DEPC- $\mathrm{H}_{2} \mathrm{O}$ and $1 \mu \mathrm{L}$ of 5 -FAM-labelled SHAPE primer (Supplementary Table 2). Primers were annealed to the template by incubation for $1 \mathrm{~min}$ at $85^{\circ} \mathrm{C}, 10 \mathrm{~min}$ at $60^{\circ} \mathrm{C}$ and $10 \mathrm{~min}$ at $35^{\circ} \mathrm{C}$. Each reaction was then combined with $1 \mu \mathrm{L}$ DTT

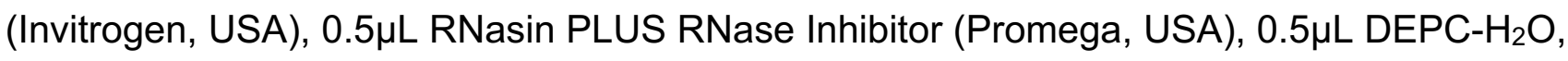
$1 \mu \mathrm{L} 10 \mathrm{mM}$ dNTPs, $1 \mu \mathrm{L}$ SuperScript III RT (Invitrogen, USA) and 4 $\mu \mathrm{L}$ SSIII First Strand Buffer (Invitrogen, USA). RT reaction was performed at $52^{\circ} \mathrm{C}$ for $30 \mathrm{~min}$ and quenched by adding $1 \mu \mathrm{L} 4 \mathrm{M} \mathrm{NaOH}$ followed by incubation at $95{ }^{\circ} \mathrm{C}$ for $3 \mathrm{~min}$. $\mathrm{NaOH}$ was neutralised by adding $2 \mu \mathrm{L}$ of $2 \mathrm{M} \mathrm{HCl}$. Reactions were subsequently chilled on ice and EtOH-precipitated overnight at $-80{ }^{\circ} \mathrm{C}$ as described above. Pelleted cDNA was dissolved in $40 \mu \mathrm{L}$ of deionised formamide at $65^{\circ} \mathrm{C}$ for $10 \mathrm{~min}$ and then stored at $-80^{\circ} \mathrm{C}$.

To prepare a sequencing ladder $10 \mu \mathrm{g}$ RNA was incubated with 10 pmol 5'-HEXlabelled SHAPE primer (Supplementary Table 2) in $10 \mu \mathrm{L}$ volume a for $1 \mathrm{~min}$ at $85^{\circ} \mathrm{C}, 10 \mathrm{~min}$ at $60{ }^{\circ} \mathrm{C}$ and $10 \mathrm{~min}$ at $35^{\circ} \mathrm{C}$. Each reaction was then combined with $1 \mu \mathrm{L}$ DTT (Invitrogen,

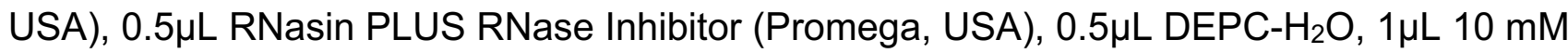
dNTPs, $1 \mu \mathrm{L}$ of $10 \mathrm{mM}$ ddTTP (Roche, USA), $1 \mu \mathrm{L}$ SuperScript III RT (Invitrogen, USA) and 
$4 \mu \mathrm{L}$ SSIII First Strand Buffer (Invitrogen, USA). The cDNA was then synthesised and precipitated as described above for the SHAPE reactions. Pelleted cDNA was dissolved in $40 \mu \mathrm{L}$ of deionised formamide at $65^{\circ} \mathrm{C}$ for $10 \mathrm{~min}$, and $10 \mu \mathrm{L}$ of the solution was combined with $40 \mu \mathrm{L}$ of (+) and (-) SHAPE cDNAs. Samples were then analysed by high throughput capillary electrophoresis. Chromatogram data was analysed, and SHAPE reactivities were computed using QuShape software ${ }^{46}$. Mean SHAPE reactivities from three independent experiments were used as constraints for guided RNA folding with RNAStructure software (https://rna.urmc.rochester.edu/RNAstructureHelp.html). The folding temperature was set to $28 \mathrm{C}$, folding of pseudoknots was enabled, and the maximum distance of base pairing was set to $100 \mathrm{nt}$. Resultant secondary structures were visualised using VARNA v3.93. Noncanonical C-A pairing in cISF sfRNAs was forced manually.

\section{RNA structure prediction}

Pseudoknots if dISF xrRNAs were predicted using IPknot ${ }^{48}$ web server (http://rtips.dna.bio.keio.ac.jp/ipknot/). Secondary structures of AnFV 3'UTR elements were predicted using mfold v2.3 (http://www.unafold.org/mfold/applications/rna-folding-formv2.php), and pseudoknots were located manually. The folding temperature was set to $28^{\circ} \mathrm{C}$, and the maximum distance between paired nucleotides was limited to 150. All secondary structures were visualised using VARNA v3.93.

\section{Multiple sequence alignment, covariance analysis, and phylogenetic inference}

Structure-based multiple alignments of xrRNAs were performed using RIBOSUM-like similarity scoring implemented in the LocARNA package (Freiburg RNA Tools) ${ }^{49}$. Experimentally determined or predicted secondary structures were provided for each xrRNAs as structural constrains (\#S option). Stockholm formatted alignments and consensus secondary structures generated by LocARNA were used to built covariance models followed by evaluation and visualisation of consensus structures with R-scape software ${ }^{37}$ 
(http://eddylab.org/R-scape/). Consensus pseudoknots were specified manually in R-scape input as consensus structures \#CS_2 and \#CS_3.

Multiple sequence alignment (MSA) used for phylogenetic inference were constructed using iterative refinement methods incorporated into MAFFT v7.47550 for both the polyprotein (G-INS-i) and complete 3'UTR (Q-INS-i; which incorporates structural information ${ }^{51}$ of 48 representative Flaviviruses. The resultant MSA dimensions were $(3897 \times 48)$ and $(1655 \times 48)$ for the polyprotein and nucleotide, respectively, and accession IDs are available from Supplementary Table 1. IQ-TREE2 (v2.1.2) ModelFinder, tree search, SH-aLRT test and ultrafast bootstrap ${ }^{52}$ were used to construct the consensus maximum-likelihood phylogenetic inference (Command: --alrt 1000 -B 1000). The best-fit protein substitution model (LG+F+R6) and nucleotide substitution model $(G T R+F+\mid+G 4)$ was selected using the ModelFinder program within IQ-TREE2 informed using the Bayesian Information Criterion. The resultant consensus tree was visualised using FigTree v1.4.4 (Rambaut, A., 2021, https://github.com/rambaut/figtree/).

\section{PCR-directed mutagenesis and generation of mutant viruses}

Plasmid constructs based on pUC19 vector containing WT 3'UTR of each ISF were used as templates for PCR-directed site-specific mutagenesis, which was performed using Q5 Site-Directed Mutagenesis Kit (NEB, USA) with mutagenesis primers listed in Supplementary Table 2. Cycling conditions for mutagenesis PCR were were $1 \mathrm{~min}$ at $98^{\circ} \mathrm{C}$; 25 cycles of $10 \mathrm{~s}$ at $98^{\circ} \mathrm{C}, 30 \mathrm{~s}$ at $55^{\circ} \mathrm{C}, 2 \mathrm{~min}$ at $72^{\circ} \mathrm{C}$; and a final extension for 2 min at 72 ${ }^{\circ} \mathrm{C}$. PCR products were analysed by electrophoresis in $1 \%$ agarose gel, and if multiple amplification products were evident, individual amplicons were gel-purified using QIAquick Spin Gel extraction kit (Qiagen, Germany). Products of PCR amplification were subjected to KLD reaction using Q5 Site-Directed Mutagenesis Kit (NEB, USA) and transformed into NEB 5-alpha Competent E. coli High Efficiency (NEB, USA) according to the manufacturer's instructions. Plasmids were isolated from $5 \mathrm{~mL}$ of overnight liquid culture using QIAprep Spin 
Miniprep Kit (Qiagen, Germany) and the presence of mutations was confirmed by Sanger Sequencing.

Mutant viruses were assembled using circular polymerase extension reaction (CPER) according to the established protocols for PaRV, PCV and BinJV. CPER fragments containing WT or mutated or 3'UTRs were amplified from the plasmids using PrimeStar GXL polymerase (Takara, Japan) and gel-purified. Infectious cDNA from CPER assembly was transfected into C6/36 cells using Lipofectamine 2000 (Invitrogen, USA) according to the manufacturer's instructions. Culture fluids of transfected cells were then sampled for virus titration at 5, 7 and 10 days post transfection. To confirm the presence of the mutations in 3'UTRs, viral RNA was isolated from culture fluid samples using QIAamp Viral RNA Kit (Qiagen, Germany) and used as a template for RT-PCR with 3'UTR sequencing primers (Supplementary table 2). RT-PCR was performed using SuperScript III One-Step RT-PCR Kit with Platinum Taq (Invitrogen, USA) and the following cycling conditions: 15 min at $60{ }^{\circ} \mathrm{C}$, 2 min at $95^{\circ} \mathrm{C} ; 35$ cycles of $15 \mathrm{~s}$ at $95^{\circ} \mathrm{C}, 30 \mathrm{~s}$ at $55^{\circ} \mathrm{C}, 40 \mathrm{sec}$ at $68^{\circ} \mathrm{C}$; and a final extension for $5 \mathrm{~min}$ at $68^{\circ} \mathrm{C}$. PCR products were separated in $2 \%$ agarose gel, purified using QIAquick Spin Gel extraction kit (Qiagen, Germany) and Sanger Sequenced.

\section{Virus growth kinetics}

The growth kinetics of wild type and mutant viruses were assessed in RML-12 cells. Cells were seeded at $2 \times 10^{6}$ cells per well in 6-well plates and inoculated with wild type or mutated viruses at an $\mathrm{MOI}$ of 0.1 by incubating for $1 \mathrm{~h}$ with $200 \mu \mathrm{L}$ of virus inoculum. Incubations were performed at $28^{\circ} \mathrm{C}$. Inoculum was then removed, and cells were washed three times with PBS and overlayed with $2 \mathrm{~mL}$ of $\mathrm{L}-15$ culture medium supplemented with $2 \%$ FCS. At time point zero, $100 \mu \mathrm{L}$ of media was immediately collected, and infected cells were then incubated for 7 days at $28^{\circ} \mathrm{C}$. Culture fluid samples $(100 \mu \mathrm{L})$ were then harvested at 3 , 5 and 7 days post-infection and subjected to foci-forming immunoassay to determine the virus titres, from which growth curves were plotted. 


\section{Statistical Analysis}

Statistical analyses were performed using GraphPad Prism v.9.0.

\section{Ethics statement}

This study was not subject to ethics approval.

\section{Data availability statement}

All data are available within the paper, supplementary material and by request from the authors.

\section{Acknowledgments}

We are grateful to Prof Jeffrey S. Kieft (University of Colorado) for providing key advice to structural aspects of the study. Work was funded by the Australian Research Council (ARC) grant DP190103304. Sanger sequencing was performed by the Australian Genomics Research Facility (Brisbane, Australia).

\section{Conflicting interests}

All authors declare no conflicting interests.

\section{Authors contributions}

AS, AAK - conceptualisation; AS - experiment design, AS, BP, JDJS, XW, TB, FJT, AT - experiments; AS, RP - data analysis, bioinformatics; JH, AMGC, JHP, RAH - critical materials and reagents; AAK, AT - project supervision; AAK, AS, RAH, JHP - funding acquisition. 
Figures and legends
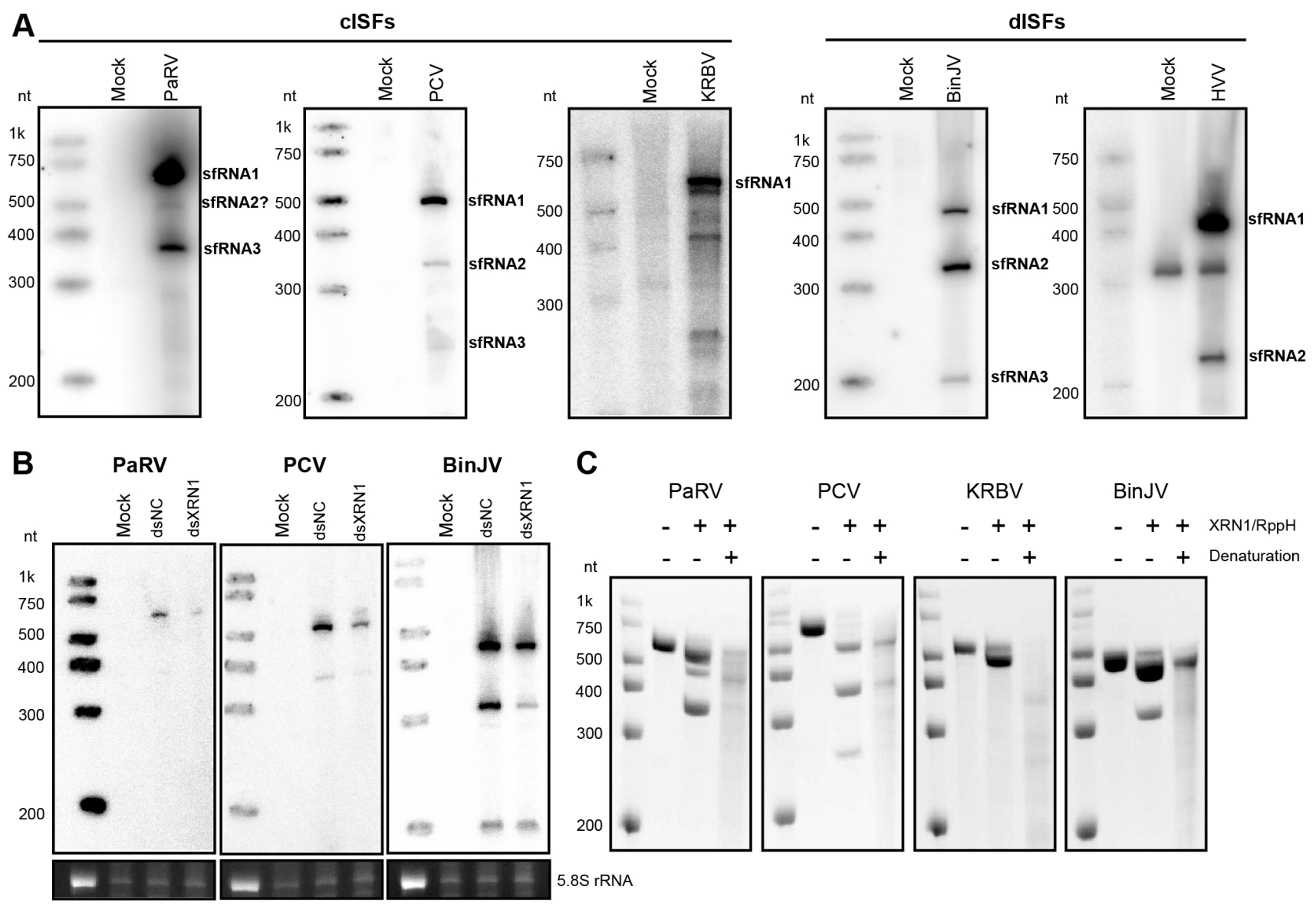

C

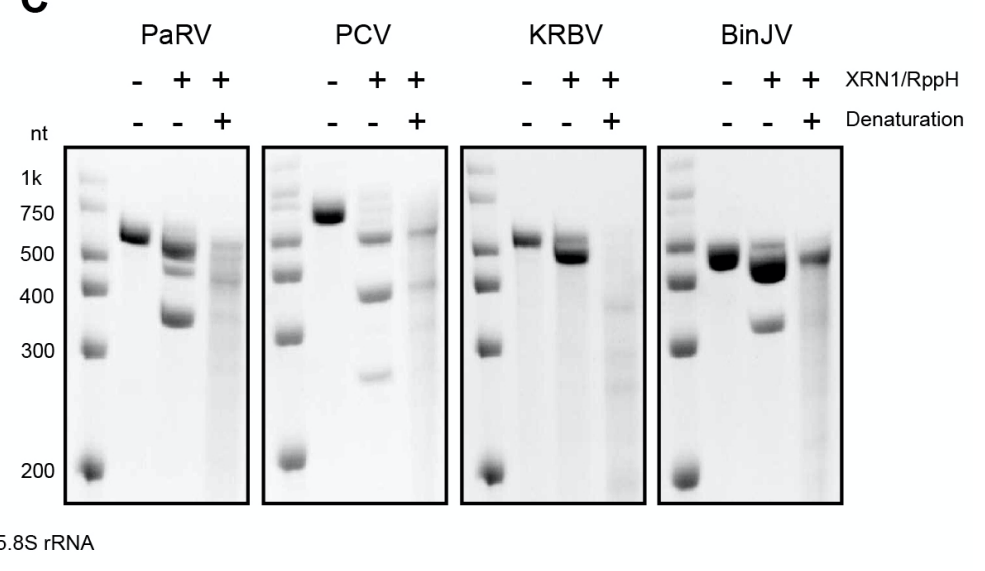

Figure 1. Classical and dual host associated ISFs produce sfRNAs employing the XRN1-resistance mechanism. (A) Northern blot detection of sfRNAs produced by ISFs. For $\mathrm{PaRV}, \mathrm{PCV}$, BinJV and HVV C6/36 cells were infected at MOI=1. Total RNA was isolated at 5 dpi. For KRBV, total RNA was isolated from virus-positive and virus-negative (Mock) Anopheles mosquitoes. RNA was then used for Northern blotting with the probe complementary to the last 25nt of viral 3'UTRs. (B) The effect of XRN-1 knock-down on the production of sfRNAs by ISFs. Aag2 cells were transfected with dsRNA against Aedes aegypti XRN-1 (dsXRN1) or GFP (dsNC) and infected with respective viruses at $\mathrm{MOl}=1$ at 24hpt. At 48hpi, total RNA was isolated from the cells and used for Northern blotting as in (A). Bottom panels represent the Et-Br staining of the gels used for Northern transfer with 5.8S rRNA visualised as an equal loading control. (C) In vitro XRN-1 resistance assay with ISF 3'UTRs. RNA corresponding to 3'UTRs of ISFs was in vitro transcribed, briefly heated and then either refolded by gradual cooling to $28 \mathrm{C}$ or placed on ice to preserve the denatured state. Samples were then treated purified XRN1 and RppH (to convert 5'PPP into 5'P) and analysed by electrophoresis in denaturing PAAG. Gels were stained with Et-Br. All images are representative of at least two independent experiments that produced similar results. 
A

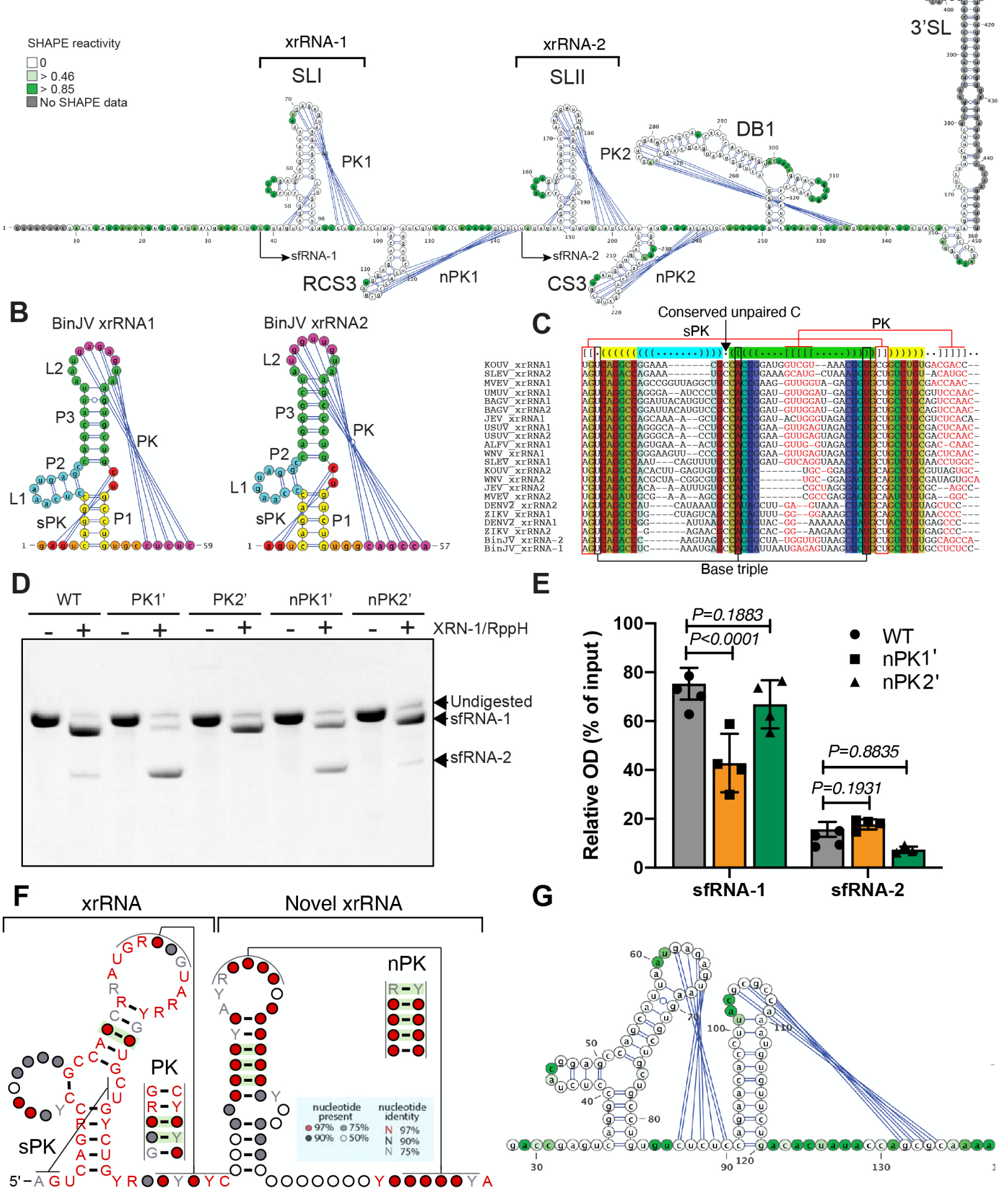

Figure 2. dISFs contain novel xrRNAs in addition to class 1a xrRNAs. (A) Secondary structure of BinJV 3'UTR generated using SHAPE-assisted folding. Colour intensity indicates NIMIA reactivity. SL - stem-loop, DB - dumbbell, RCS3 - reverse conserved sequence 3, CS3 - conserved sequence 3, PK - pseudoknot, nPK - novel pseudoknot. (B) Secondary structures of BinJV SL. Colour coding shows the location of each structural element. (C) Structure-based sequence alignment between BinJV xrRNAs and MBF xrRNAs. Conserved structural elements and interactions required for folding into a ring-like conformation are shown. Colour legend is the same as in Supplementary figure 3C. (D) In vitro XRN-1 resistance assay with WT and mutated BinJV 3'UTRs. PK1', PK2', nPK1' and nPK2' mutations were introduced into the terminal loop regions of SLI, SLII, RCS3 and CS3 and 
represented the substitutions of all nucleotides involved in PK interactions with complementary nucleotides. The image is representative of four independent experiments that produced similar results. (E) Gel densitometry analysis of (C) showing the effects of mutations in novel pseudoknots on the production of BinJV sfRNAs. The values are the means of four independent experiments \pm SD. Statistical analysis was performed by one-way ANOVA. (F) Consensus structure of dISF xrRNAs built based on the covariance model. Covarying base pairs are highlighted in green shading, $R-A / G, Y-C / U$. (G) Secondary structure of HVV xrRNA and novel xrRNA generated using SHAPE-assisted folding. The colour key for NIMIA reactivity is as in (A). SHAPE reactivity values in $(A)$ and $(G)$ are the means from 3 independent experiment, each with technical triplicates. 


\section{A}
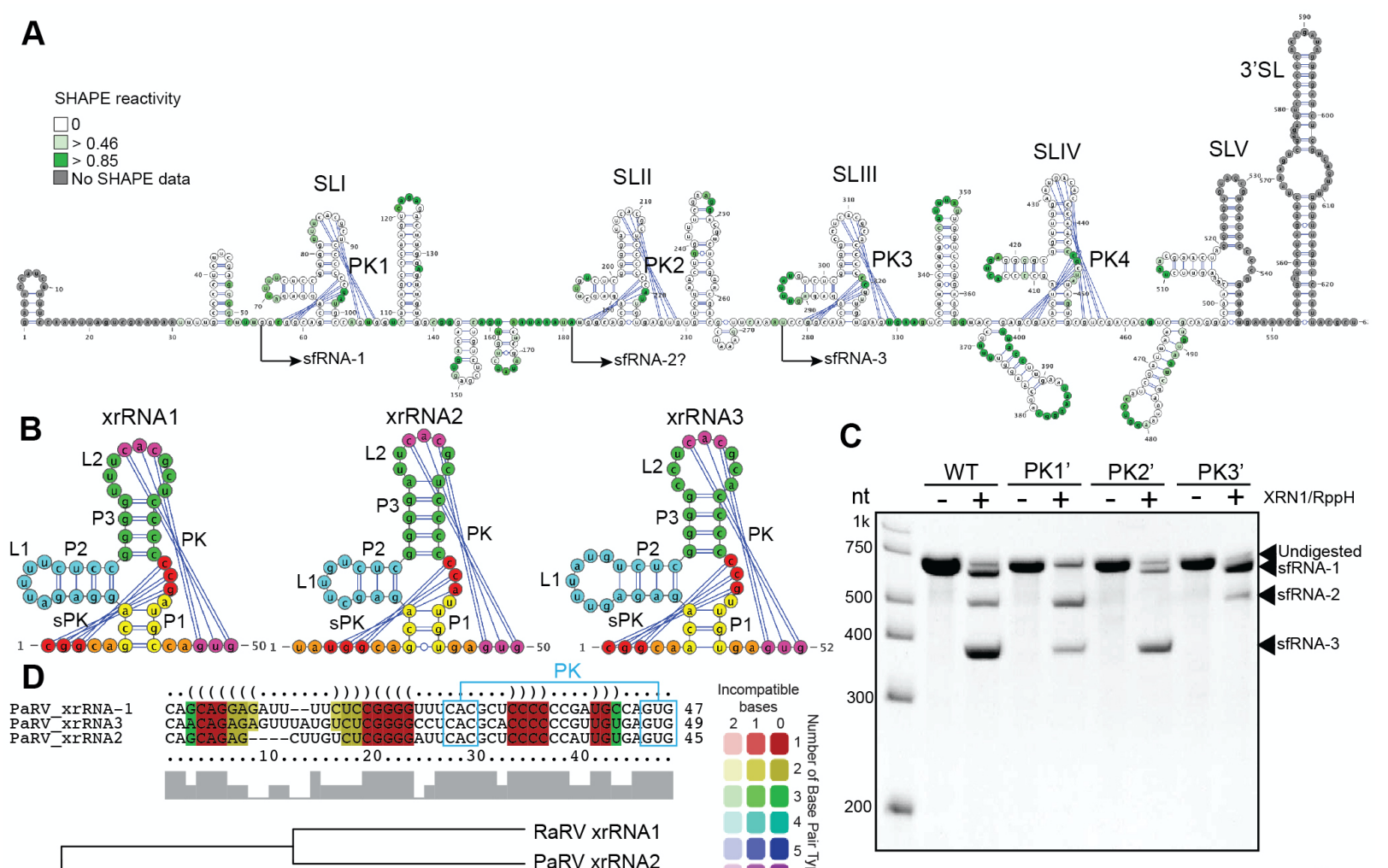

D
PaRV_XrRNA-1
PaRV_XrRNA3
PaRV_XrRNA2
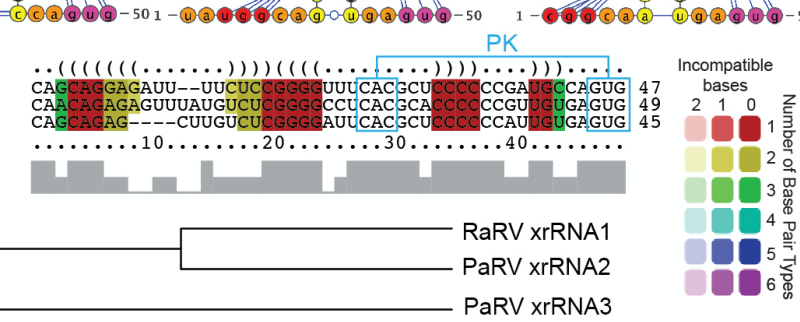

SHAPE reactivity
$\square 0$
$\square>0.46$
$\nabla 0.85$
No SHAPE data

SLI

SLII

SLIII
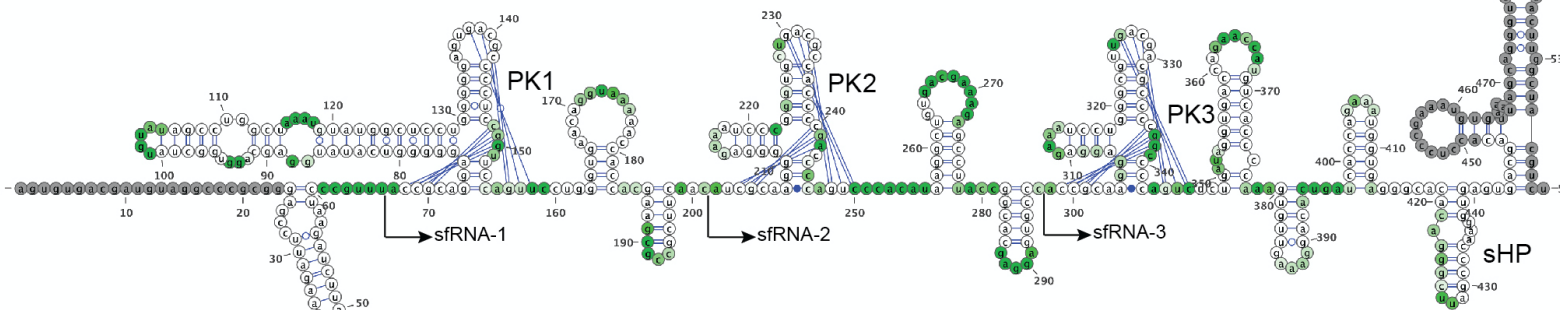

$\mathbf{F}$

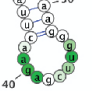

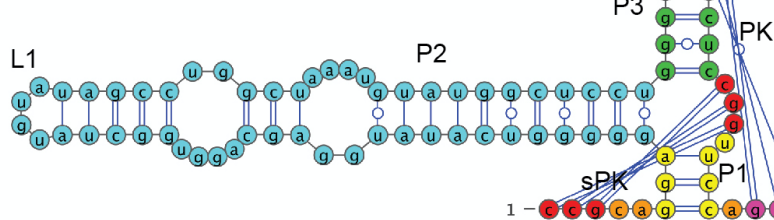

1 -ccaca-g=(c) PK

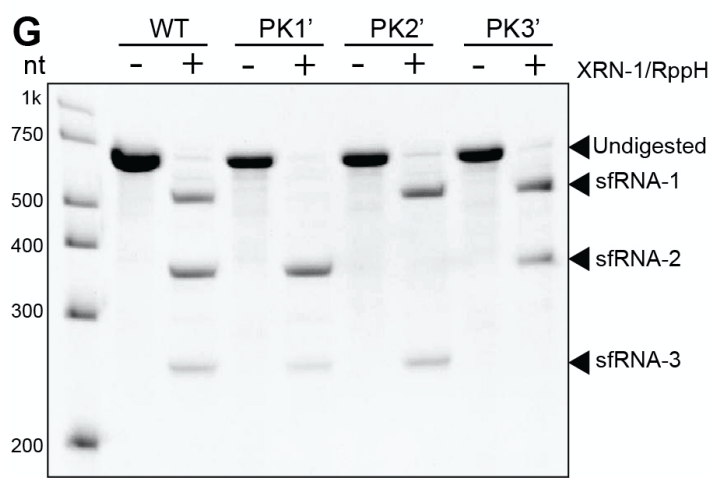

H

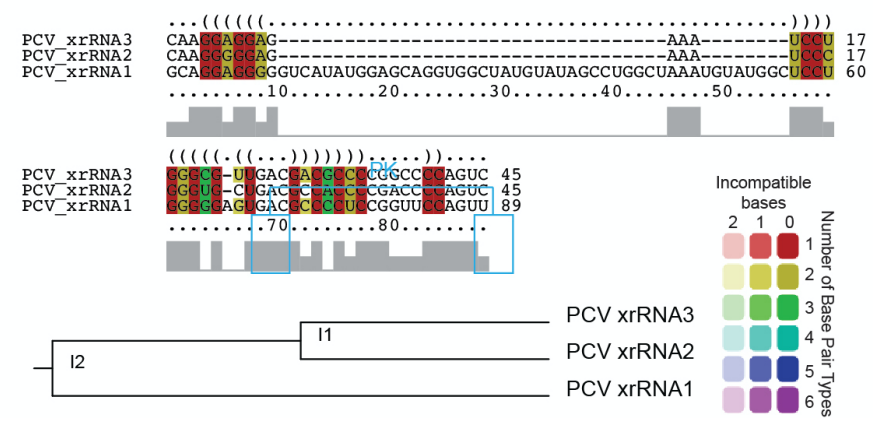


Figure 3. cISFs PaRV and PCV contain multiple copies of divergent class $1 \mathrm{~b}$ xrRNAs. (A) Secondary structure of PaRV 3'UTR generated using SHAPE-assisted folding. Colour intensity indicates NIMIA reactivity. Values are the means from 3 independent experiment, each with technical triplicates. SL - stem loop, PK -pseudoknot. (B) Secondary structure of $\mathrm{PaRV}$ xrRNAs. Each structural element is shown in colour. (C) In vitro XRN-1 resistance assay with WT and mutated PaRV 3'UTRs. PK1', PK2' and PK3' mutations were introduced into the terminal loop regions of SLI, SLII and SLIII, respectively, and represented the CAC $>$ GUG change in the PK-forming region. (D) Structure-based alignment of PaRV xrRNAs performed using LocARNA. (E) Secondary structure of PCV 3'UTR generated using SHAPEassisted folding. Shading intensity indicates NIMIA reactivity. Values are the means from 3 independent experiment, each with technical triplicates. SL - stem loop, PK - pseudoknot. (F) Secondary structure of PCV xrRNAs. Each element of the secondary structure is shown in colour. (G) In vitro XRN-1 resistance assay with WT and mutated PCV 3'UTRs. PK1', PK2' and PK3' mutations were introduced into the terminal loop regions of SLI, SLII and SLIII, respectively, and represented the GAC -> CUG change in the PK-forming region. $(\mathbf{H})$ Alignment of sequence and structure of PCV xrRNAs performed using LocARNA.

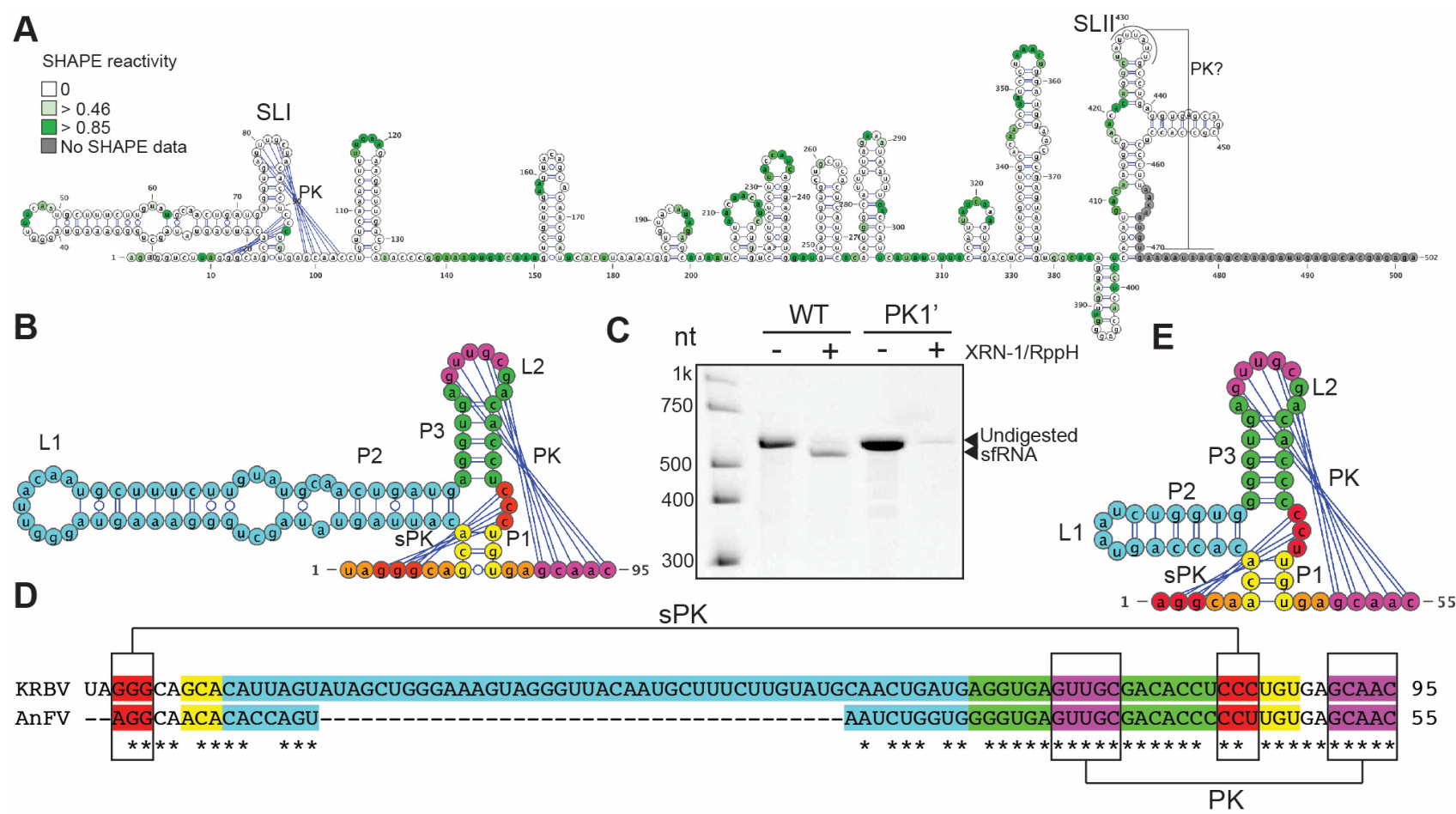

Figure 4. Anopheles associated ISFs contain a single copy of xrRNA, which shares features of class 1a and class 1b xrRNAs. (A) Secondary structure of KRBV 3'UTR generated using SHAPE-assisted folding. Shading intensity indicates NIMIA reactivity. Values are the means from 3 independent experiment, each with technical triplicates. SL stem loop, PK -pseudoknot. (B) Secondary structure of KRBV xrRNA. Each element of the secondary structure is shown in colour. (C) In vitro $\mathrm{XRN}-1$ resistance assay with WT and mutated KRBV 3'UTRs. PK1' mutation was GUUGC -> CAACG change of PK-forming nucleotides in the L2 loop of the SLI. (D) Sequence alignment of KRBV and AnFV1 xrRNAs. The colour coding matches (B) and shows conserved structural elements. (E) Predicted secondary structure of AnFV1 xrRNA. The conserved structural elements are shown in colours that match (D). 

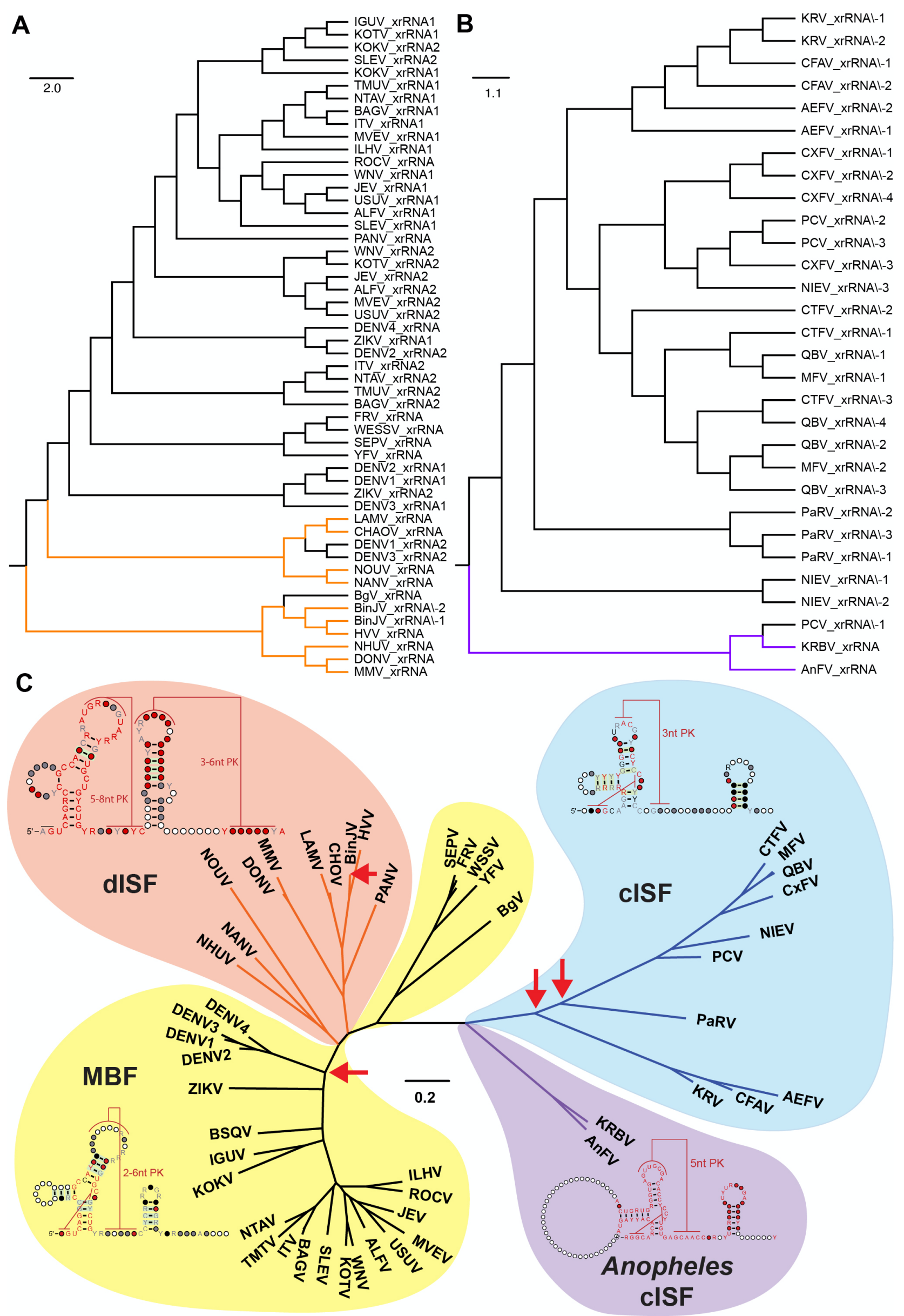

Figure 5. Phylogenetic analysis of flavivirus xrRNAs and 3'UTRS. (A) Tree of phylogenetic relationships of cISF xrRNAs obtained based on the structural alignment. 
Anopheles-associated ISFs are shown in purple. (B) Tree of phylogenetic relationships of MBF and dISF xrRNAs obtained based on the structural alignment. dISF clades are shown in orange. In (A, B), alignments were performed and distances computed using the LocARNA package, for which secondary structures of xrRNAs were specified as structural constraints. Experimentally determined secondary structures from this or previous studies were used if possible; otherwise, predicted secondary structures published in the literature or determined in this study were utilised. (C) Consensus Maximum-Likelihood phylogenetic tree of flavivirus 3'UTRs aligned using sequence and structural information and consensus xrRNA structures. Red arrows indicate xrRNA duplication events. Bootstrap supports for the nodes are shown in Supplementary figure 6. Consensus structures were generated using covariance models based on alignments with xrRNA structures determined in this study (dISFs, clSFs) or published previously (MBFs).
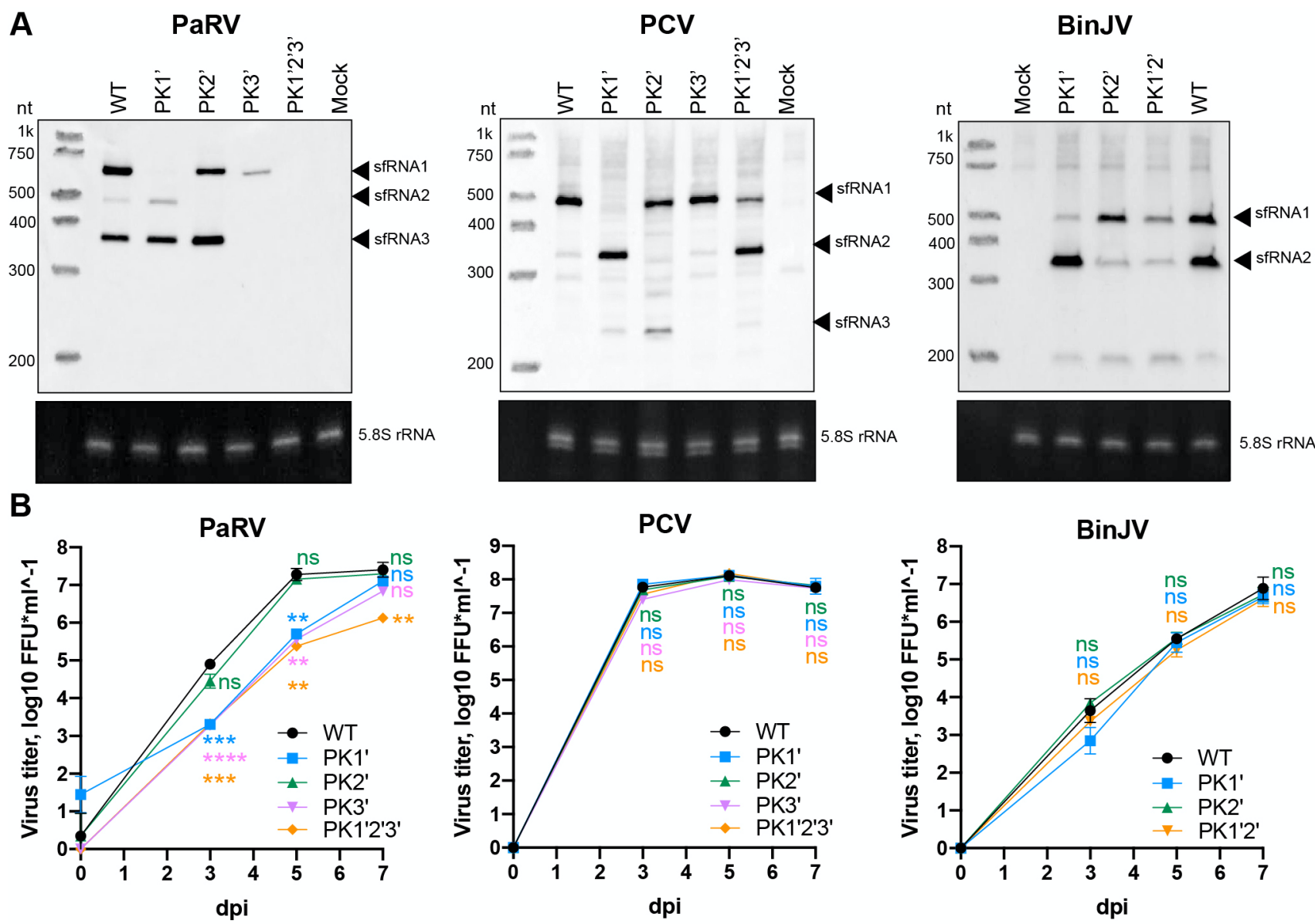

Figure 6. Effect of sfRNA deficiency on replication of ISFs in mosquito cells. (A) Northern blot analysis of sfRNA production by WT and PK mutants of PARV, PCV and BinJV. $\mathrm{C} 6 / 36$ cells were infected with WT or mutant viruses at $\mathrm{MOI}=1$. Total RNA was isolated at 7 dpi and used for blotting. The bottom panel show Et-Br-stained 5.8S rRNA as an equal loading control. Images are representative of two independent experiments that produced similar results. PK1', PK2, and PK3' mutations represented substitutions of PK-forming nucleotides in L2 loops of SLI, SLII and SLII, respectively, with complementary nucleotides. (B) Virus titers in culture fluids of RML-12 cells infected with WT or PK-mutants of ISFs. Cells were infected at $\mathrm{MOI}=0.1$, culture fluids were sampled the indicated time point, and titers were determined using foci-forming immunoassay on C6/36 cells. Values are the means from three biological replicates $\pm S D$. Statistical analysis was performed by two-way ANOVA with Dunnett's correction, all comparisons were to WT; ${ }^{\star} P<0.05,{ }^{* \star} P<0.01,{ }^{* \star *} P<0.001$, ${ }^{* * * *} P<0.0001$, ns - not significant. 


\section{References}

1. Laureti, M., Paradkar, P. N., Fazakerley, J. K. \& Rodriguez-Andres, J. Superinfection exclusion in mosquitoes and its potential as an arbovirus control strategy. Viruses 12, $1-17$ (2020).

2. Hobson-Peters, J. et al. A recombinant platform for flavivirus vaccines and diagnostics using chimeras of a new insect-specific virus. Sci. Transl. Med. 11, 1-16 (2019).

3. LaSala, P. R. \& Holbrook, M. Tick-borne flaviviruses. Clin. Lab. Med. 30, 221-235 (2010).

4. Blitvich, B. J. \& Firth, A. E. A Review of flaviviruses that have no known arthropod vector. Viruses 9, 154 (2017).

5. Blitvich, B. J. \& Firth, A. E. Insect-specific flaviviruses: A systematic review of their discovery, host range, mode of transmission, superinfection exclusion potential and genomic organization. Viruses 7, (2015).

6. Hall, R. A. et al. Commensal viruses of mosquitoes: Host restriction, transmission, and interaction with arboviral pathogens. Evol. Bioinforma. 12s2, EBO.S40740 (2016).

7. Slonchak, A. \& Khromykh, A. A. Subgenomic flaviviral RNAs: What do we know after the first decade of research. Antiviral Res. 159, 13-25 (2018).

8. Nagarajan, V. K. XRN 5' $\rightarrow$ ' exoribonucleases: Structure, mechanisms and functions. Biochim Biophys Acta 1829, 590-603 (2013).

9. Funk, A. et al. RNA structures required for production of subgenomic flavivirus RNA. J. Virol. 84, 11407-17 (2010).

10. Pijlman, G. P. et al. A highly structured, nuclease-resistant, noncoding RNA produced by flaviviruses is required for pathogenicity. Cell Host Microbe 4, 579-591 (2008).

11. Slonchak, A., et al. Zika virus noncoding RNA cooperates with the viral protein NS5 
to inhibit STAT1 phosphorylation and facilitate viral pathogenesis. bioRxiv (2021). doi:10.1101/2021.05.18.444753

12. Schuessler, a. et al. West Nile virus noncoding subgenomic RNA contributes to viral evasion of the type I interferon-mediated antiviral response. J. Virol. 86, 5708-5718 (2012).

13. Filomatori, C. V. et al. Dengue virus genomic variation associated with mosquito adaptation defines the pattern of viral non-coding RNAs and fitness in human cells. PLoS Pathog. 13, 1-23 (2017).

14. Manokaran, G. et al. Dengue subgenomic RNA binds TRIM25 to inhibit interferon expression for epidemiological fitness. Science. 350, 217-221 (2015).

15. Bidet, K., Dadlani, D. \& Garcia-Blanco, M. A. G3BP1, G3BP2 and CAPRIN1 are required for translation of interferon stimulated mRNAs and are targeted by a Dengue virus non-coding RNA. PLoS Pathog. 10, (2014).

16. Schnettler, E. et al. Noncoding flavivirus RNA displays RNA interference suppressor activity in insect and mammalian cells. J. Virol. 86, 13486-13500 (2012).

17. Schnettler, E. et al. Induction and suppression of tick cell antiviral RNAi responses by tick-borne flaviviruses. Nucleic Acids Res. 42, 9436-9446 (2014).

18. Slonchak, A. et al. Zika virus noncoding RNA suppresses apoptosis and is required for virus transmission by mosquitoes. Nat. Commun. 11, 1-14 (2020).

19. MacFadden, A. et al. Mechanism and structural diversity of exoribonuclease-resistant RNA structures in flaviviral RNAs. Nat. Commun. 9, 119 (2018).

20. Akiyama, B. M. et al. Zika virus produces noncoding RNAs using a multi-pseudoknot structure that confounds a cellular exonuclease. Science. 354, 1148-1152 (2016).

21. Chapman, E. G. et al. The structural basis of pathogenic subgenomic flavivirus RNA (sfRNA) production. Science. 344, 307-310 (2014). 
22. Kieft, J. S., Rabe, J. L. \& Chapman, E. G. New hypotheses derived from the structure of a flaviviral Xrn1-resistant RNA: Conservation, folding, and host adaptation. RNA Biol. 12, 1117-1169 (2015).

23. Chapman, E. G., Moon, S. L., Wilusz, J. \& Kieft, J. S. RNA structures that resist degradation by Xrn1 produce a pathogenic Dengue virus RNA. eLife 3, 1-25 (2014).

24. Silva, P. A. G. C., Pereira, C. F., Dalebout, T. J., Spaan, W. J. M. \& Bredenbeek, P. J. An RNA pseudoknot is required for production of Yellow Fever virus subgenomic RNA by the host nuclease XRN1. J. Virol. 84, 11395-11406 (2010).

25. Jones, R. A. et al. Different tertiary interactions create the same important 3D features in a distinct flavivirus xrRNA. RNA. 27, 54-65 (2021).

26. Steckelberg, A.-L., Vicens, Q. \& Kieft, J. S. Exoribonuclease-resistant RNAs exist within both coding and noncoding subgenomic RNAs. MBio 9, e02461-18 (2018).

27. Villordo, S. M., Carballeda, J. M., Filomatori, C. V. \& Gamarnik, A. V. RNA structure duplications and flavivirus host adaptation. Trends Microbiol. 24, 270-283 (2016).

28. Clarke, B. D., Roby, J. A., Slonchak, A. \& Khromykh, A. A. Functional non-coding RNAs derived from the flavivirus 3' untranslated region. Virus Res. 206, 53-61 (2015).

29. Akiyama, B. M., Graham, M. E., O'donoghue, Z., David Beckham, J. \& Kieft, J. S. Three-dimensional structure of a flavivirus dumbbell RNA reveals molecular details of an RNA regulator of replication. Nucleic Acids Res Jun 16;gkab462. doi: 10.1093/nar/gkab462 (2021)

30. Gritsun, D. J. et al. Molecular archaeology of Flaviviridae untranslated regions: Duplicated RNA structures in the replication enhancer of Flaviviruses and Pestiviruses emerged via convergent evolution. PLoS One 9, 1-11 (2014).

31. McLean, B. J. et al. A novel insect-specific flavivirus replicates only in Aedes-derived 
cells and persists at high prevalence in wild Aedes vigilax populations in Sydney, Australia. Virology 486, 272-283 (2015).

32. Hobson-Peters, J. et al. A new insect-specific flavivirus from Northern Australia suppresses replication of West Nile virus and Murray Valley encephalitis virus in coinfected mosquito cells. PLoS One 8, 1-12 (2013).

33. Harrison, J.J. et al. Antigenic Characterization of new lineage II insect-specific flaviviruses in Australian mosquitoes and identification of host restriction factors. mSphere 5, e00095-20 (2020).

34. Colmant, A. M. G. et al. A new clade of insect-specific flaviviruses from Australian Anopheles mosquitoes displays species-specific host restriction. mSphere 2, e0026217 (2017).

35. Deigan, K. E., Li, T. W., Mathews, D. H. \& Weeks, K. M. Accurate SHAPE-directed RNA structure determination. Proc. Natl. Acad. Sci. U. S. A. 106, 97-102 (2009).

36. Sato, K., Kato, Y., Hamada, M., Akutsu, T. \& Asai, K. IPknot: fast and accurate prediction of RNA secondary structures with pseudoknots using integer programming. Bioinformatics 27, i85-i93 (2011).

37. Rivas, E., Clements, J. \& Eddy, S. R. A statistical test for conserved RNA structure shows lack of evidence for structure in IncRNAs. Nat. Methods 14, 45-48 (2016).

38. Villordo, S. M., Filomatori, C. V., Sánchez-Vargas, I., Blair, C. D. \& Gamarnik, A. V. Dengue Virus RNA Structure Specialization Facilitates Host Adaptation. PLoS Pathog. 11, 1-22 (2015).

39. Finol, E. \& Ooi, E. E. Evolution of Subgenomic RNA Shapes Dengue Virus Adaptation and Epidemiological Fitness. iScience 16, 94-105 (2019).

40. Göertz, G. P. et al. Subgenomic flavivirus RNA binds the mosquito DEAD / H-box helicase ME31B and determines Zika virus transmission by Aedes aegypti. Proc. 
Natl. Acad. Sci. U. S. A. 116, 19136-19144 (2019).

41. Göertz, G. P. et al. Noncoding Subgenomic Flavivirus RNA Is Processed by the Mosquito RNA Interference Machinery and Determines West Nile Virus Transmission by Culex pipiens Mosquitoes. J. Virol. 90, 10145-10159 (2016).

42. Steckelberg, A.-L. et al. A folded viral noncoding RNA blocks host cell exoribonucleases through a conformationally dynamic RNA structure. Proc. Natl. Acad. Sci. 115, 6404-6409 (2018).

43. Kuno, G. Cultivation of mosquito cell lines in serum-free media and their effects on dengue virus replication. In Vitro 19, 707-713 (1983).

44. Piyasena, T. B. H. et al. Infectious DNAs derived from insect-specific flavivirus genomes enable identification of pre- and post-entry host restrictions in vertebrate cells. Sci. Rep. 7, 2940 (2017).

45. Slonchak, A., Shannon, R. P., Pali, G. \& Khromykh, A. A. Human MicroRNA miR532-5p Exhibits Antiviral Activity against West Nile Virus via Suppression of Host Genes SESTD1 and TAB3 Required for Virus Replication. J. Virol. 90, 2388-2402 (2015).

46. Karabiber, F. et al. QuShape: Rapid, accurate, and best-practices quantification of nucleic acid probing information, resolved by capillary electrophoresis. $R N A 19,63-$ 73 (2013).

47. Popenda, M. et al. Automated 3D structure composition for large RNAs. Nucleic Acids Res. 40, 1-12 (2012).

48. Sato, K., Kato, Y., Hamada, M., Akutsu, T. \& Asai, K. IPknot: Fast and accurate prediction of RNA secondary structures with pseudoknots using integer programming. Bioinformatics 27, 85-93 (2011).

49. Will, S., Joshi, T., Hofacker, I. L., Stadler, P. F. \& Backofen, R. LocARNA-P: Accurate 
boundary prediction and improved detection of structural RNAs. RNA 18, 900-914 (2012).

50. Katoh, K. \& Standley, D. M. MAFFT multiple sequence alignment software version 7: Improvements in performance and usability. Mol. Biol. Evol. 30, 772-780 (2013).

51. Katoh, K. \& Toh, H. Improved accuracy of multiple ncRNA alignment by incorporating structural information into a MAFFT-based framework. BMC Bioinformatics 9, 1-13 (2008).

52. Minh, B. Q. et al. IQ-TREE 2: New Models and Efficient Methods for Phylogenetic Inference in the Genomic Era. Mol. Biol. Evol. 37, 1530-1534 (2020). 